\title{
An Extensive \\ Pre-Cretaceous Weathering Profile in \\ East-Central and Southwestern Minnesota
}

\section{U.S. GEOLOGICAL SURVEY BULLETIN 1989-H}

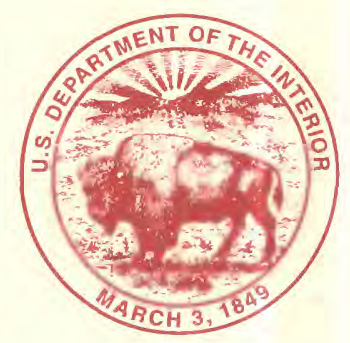




\section{AVAILABILITY OF BOOKS AND MAPS OF THE U.S. GEOLOGICAL SURVEY}

Instructions on ordering publications of the U.S. Geological Survey, along with prices of the last offerings, are given in the current-year issues of the monthly catalog "New Publications of the U.S. Geological Survey." Prices of available U.S. Geological Survey publications released prior to the current year are listed in the most recent annual "Price and Availability List." Publications that may be listed in various U.S. Geological Survey catalogs (see back inside cover) but not listed in the most recent annual "Price and Availability List" may no longer be available.

Reports released through the NTIS may be obtained by writing to the National Technical Information Service, U.S. Department of Commerce, Springfield, VA 22161; please include NTIS report number with inquiry.

Order U.S. Geological Survey publications by mail or over the counter from the offices listed below.

BY MAIL

\section{Books}

Professional Papers, Bulletins, Water-Supply Papers, Techniques of Water-Resources Investigations, Circulars, publications of general interest (such as leaflets, pamphlets, booklets), single copies of Earthquakes \& Volcanoes, Preliminary Determination of Epicenters, and some miscellaneous reports, including some of the foregoing series that have gone out of print at the Superintendent of Documents, are obtainable by mail from

\section{U.S. Geological Survey, Information Services Box 25286, Federal Center Denver, CO 80225}

Subscriptions to periodicals (Earthquakes \& Volcanoes and Preliminary Determination of Epicenters) can be obtained ONLY from the

\section{Superintendent of Documents Government Printing Office Washington, DC 20402}

(Check or money order must be payable to Superintendent of Documents.)

\section{Maps}

For maps, address mail orders to

\section{U.S. Geological Survey, Information Services Box 25286, Federal Center Denver, CO 80225}

Residents of Alaska may order maps from

\author{
U.S. Geological Survey, Earth Science Information Center \\ 101 Twelfth Ave., Box 12 \\ Fairbanks, AK 99701
}

\section{OVER THE COUNTER}

\section{Books and Maps}

Books and maps of the U.S. Geological Survey are available over the counter at the following U.S. Geological Survey offices, all of which are authorized agents of the Superintendent of Documents.

- ANCHORAGE, Alaska-Rm. 101, 4230 University Dr.

- LAKEWOOD, Colorado-Federal Center, Bldg. 810

- MENLO PARK, California-BIdg. 3, Rm. 3128, 345 Middlefield $\mathrm{Rd}$.

- RESTON, Virginia-USGS National Center, Rm. 1C402, 12201 Sunrise Valley Dr.

- SALT LAKE CITY, Utah-Federal Bldg., Rm. 8105 , 125 South State St.

- SPOKANE, Washington-U.S. Post Office Bldg., Rm. 135, West 904 Riverside Ave.

- WASHINGTON, D.C.-Main Interior Bldg., Rm. 2650, 18th and C Sts., NW.

\section{Maps Only}

Maps may be purchased over the counter at the following U.S. Geological Survey offices:

- FAIRBANKS, Alaska-New Federal Bldg, 101 Twelfth Ave.

- ROLLA, Missouri-1400 Independence Rd.

- STENNIS SPACE CENTER, Mississippi-BIdg. 3101 
Chapter $\mathrm{H}$

\section{An Extensive \\ Pre-Cretaceous Weathering Profile in East-Central and Southwestern Minnesota}

By Dale R. Setterholm and G.B. Morey

U.S. GEOLOGICAL SURVEY BULLETIN 1989

STRATEGIC AND CRITICAL MINERALS IN THE MIDCONTINENT REGION, UNITED STATES 


\title{
U.S. DEPARTMENT OF THE INTERIOR BRUCE BABBITT, Secretary
}

\author{
U.S. GEOLOGICAL SURVEY \\ Gordon P. Eaton, Director
}

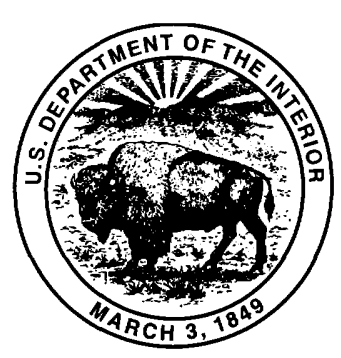

Published in the Central Region, Denver, Colo.

Manuscript approved for publication May 18, 1995.

Photocomposition by Carol A. Quesenberry.

Any use of trade, product, or firm names in this publication is for descriptive purposes only and does not imply endorsement by the U.S. Government.

UNITED STATES GOVERNMENT PRINTING OFFICE: 1995

For sale by

U.S. Geological Survey, Information Services

Box 25286, Federal Center

Denver, CO 80225

\section{Library of Congress Cataloging-in-Publication Data}

Setterholm, Dale R.

An extensive pre-Cretaceous weathering profile in east-central and southwestern Minnesota / by Dale R. Setterholm and G.B. Morey. p. cm.-(U.S. Geological Survey bulletin ; 1989)

(Strategic and critical minerals in the midcontinent region, United States ; ch. H)

Includes bibliographical references.

Supt. of Docs. no. : I $19.3: 1989-\mathrm{H}$

1. Weathering-Minnesota. 2. Saprolites-Minnesota.

I. Morey, G.B. II. Title. III. Series. IV. Series : Strategic and critical minerals in the midcontinent region, United States; ch. $\mathrm{H}$. QE75.B9 no. 1989-H

[QE570]

$557.3 \mathrm{~s}-\mathrm{dc} 20$

[551.3'02'09776] 95-30813 


\title{
CONTENTS
}

\author{
Abstract $\quad$ H1 \\ Introduction $\quad$ H1 \\ Methodology H2 \\ Mesozoic paleogeography $\quad$ H3 \\ General description of the weathering profiles H6 \\ Discussion H9 \\ Mineralogical attributes of the model $\quad$ H9

Slightly weathered zone H9
Saprock H9
Saprolith H10
Pisolitic lateritic clay $\mathbf{H 2 3}$
mical attributes of the model \\ Chemical attributes of the model \\ Summary H 26 \\ Economic implications $\quad \mathbf{H 2 7}$ \\ References cited $\mathbf{H 2 9}$
}

FIGURES

H1. Index map of Minnesota showing the approximate distribution of saprolith and associated weathering products

H2

H2. Map of the St. Cloud area, east-central Minnesota, showing the locations of drill holes discussed in the text

$\mathrm{H} 2$

H3. Map of the Redwood Falls-Morgan area along the Minnesota River Valley showing the locations of drill holes discussed in the text

H3

H4. Representative X-ray diffraction diagrams of the $<2 \mu$ m clay fraction from the St. Augusta site in east-central Minnesota

\section{H4}

H5-H12. Summary of the principal facts regarding the weathering profile at the:

H5. Cold Spring site H10

H6. St. Joseph site H11

H7. St. Augusta site $\mathbf{H 1 2}$

H8. Redwood Falls 1 site $\quad \mathbf{H 1 3}$

H9. Redwood Falls 2 site $\quad$ H14

H10. Redwood Falls 3 site $\quad$ H15

H11. Morgan 1 site $\mathbf{H 1 5}$

H12. Morgan 2 site $\mathbf{H 1 6}$

H13-H15. Whole-rock chemical trends at the:

H13. St. Cloud area sites $\quad$ H17

H14. Redwood Falls sites $\mathbf{H 1 8}$

H15. Morgan sites H19

H16-H18. Chemical characteristics of the:

H16. St. Cloud area weathering profiles $\quad$ H20

H17. Redwood Falls weathering profiles $\mathbf{H 2 1}$

H18. Morgan 1 and Morgan 2 weathering profiles $\quad \mathbf{H 2 2}$

H19. Plots of modal proportions of mineral constituents in samples, ordered by CIA values

H24 
H20. Plots of relative proportions of clay-sized material versus CIA $\quad \mathbf{H 2 6}$

$\mathrm{H} 21$. Ternary diagrams in the $\mathrm{Al}_{2} \mathrm{O}_{3}-\mathrm{CaO}+\mathrm{Na}_{2} \mathrm{O}-\mathrm{K}_{2} \mathrm{O}$ and $\mathrm{Al}_{2} \mathrm{O}_{3}-\mathrm{CaO}+\mathrm{Na}_{2} \mathrm{O}+\mathrm{K}_{2} \mathrm{O}-\mathrm{FeO}+\mathrm{MgO}$ fields showing chemical trends in the weathering of granitic rocks

$\mathrm{H} 28$

H22. Physical stratigraphy of the in-situ and reworked weathering products

H29

\section{TABLES}

H1. Chemical analysis of saprolith and associated weathering products

H2. Nature of protolith at St. Cloud area sites

H9

H3. Nature of protolith at Redwood Falls area sites

H9 


\title{
An Extensive Pre-Cretaceous Weathering Profile in East-Central and Southwestern Minnesota
}

\author{
By Dale R. Setterholm¹ and G.B. Morey ${ }^{1}$
}

\begin{abstract}
A thick mantle of residual weathering products covers crystalline bedrock of Archean to Early Proterozoic age in much of western Minnesota. Within the residuum there is a typical sequence of four lithologic units representing progressively more weathered material upward from the fresh bedrock surface. From bottom to top, they are (1) a zone of very slightly weathered bedrock that exhibits very few visible effects of weathering; (2) saprock, or weathered bedrock that is firm but distinctly altered; (3) saprolith that is typically so weak it can easily be crushed by hand but that mostly retains the structures and textures of the parent rock; and (4) a hard lateritic clay characterized by iron oxide-rich pisolites. This sequence may be partly eroded and (or) overlain by Cretaceous or Quaternary deposits. In some places the Cretaceous deposits consist of relatively pure kaolinitic shale derived from the reworking of the pre-Cretaceous saprolith.
\end{abstract}

Because the composition of these weathering products is, in part, controlled by the composition of the parent rock, heterogeneity in the parent rock typically persists as heterogeneity in the composition of the weathering profiles. For example, amphibolite rafts within a granitic gneiss result in pods of chloritic saprolith within predominantly kaolinitic saprolith. Fracture sets, joints, cleavage planes, and schistosity also exert a strong influence on the path and rate of chemical weathering and lead to anisotropic weathering patterns and complex mineral distribution patterns.

In spite of many complexities it is possible to distinguish an orderly sequence of chemical processes that can be related to the stages of weathering described above. Three main trends are apparent: (1) loss of $\mathrm{CaO}, \mathrm{Na}_{2} \mathrm{O}, \mathrm{K}_{2} \mathrm{O}$, and some $\mathrm{SiO}_{2} ;(2)$ gain in carbon dioxide; and (3) relative gains or no change in $\mathrm{Fe}_{2} \mathrm{O}_{3}, \mathrm{MgO}, \mathrm{TiO}_{2}, \mathrm{Al}_{2} \mathrm{O}_{3}$, and water content. These chemi$\mathrm{cal}$ changes are consistent with the ferrallitic weathering trend (Chesworth, 1992) characteristic of stable landscapes in the humid tropics. The correlation of chemical alteration indices with modal mineralogy makes it apparent that the loss of $\mathrm{Na}_{2} \mathrm{O}, \mathrm{CaO}, \mathrm{K}_{2} \mathrm{O}$, and possibly some silica corresponds with the progressive disappearance of first plagioclase, and then

${ }^{1}$ Minnesota Geological Survey, University of Minnesota, St. Paul, MN 55114-1057. potassium feldspar. With continued weathering more potassium is lost as potassium feldspar continues to dissolve. Total iron content is more or less constant in the lower two-thirds, but increases appreciably in the upper third of most profiles. This probably reflects the breakdown of hornblende and biotite and the concurrent release of ferrous iron, which migrates toward the upper part of the profile, where oxidizing conditions are encountered and it is fixed as a ferric oxide. Diagenetic modifications of these weathering processes typically add $\mathrm{SiO}_{2}, \mathrm{CO}_{2}, \mathrm{Fe}_{2} \mathrm{O}_{3}$, and $\mathrm{MgO}$ to the upper parts of weathering profiles, where they form oxides, carbonates, or clay minerals such as smectites. Potential users of kaolinite require a deposit that is thick, widespread, rich in kaolin, and relatively free of impurities that would tend to color the deposit. The upper part of the saprolitic interval where the most intense chemical weathering has occurred best meets these needs. However, diagenetic changes are also most likely to occur in this zone and the user must expect that at least some beneficiation will be necessary.

Natural gamma logging is a quick and inexpensive means of recognizing weathering products, evaluating the degree of weathering, and measuring the extent of the deposit. In weathered terranes the predominant clay mineral is kaolinite, and because it contains no potassium (or uranium or thorium), the parts of the profile richest in kaolinitic clay have the lowest gamma values. In general, gamma values increase with depth through the saprolith and saprock and into the protolith. The effects of uranium and thorium are typically confined to gamma peaks associated with veining in the protolith or bauxitic intervals.

\section{INTRODUCTION}

A thick mantle of saprolith and associated weathering products covers crystalline bedrock of Archean and Proterozoic age in much of central and western Minnesota (fig. H1). The profile is typically more weathered upward from fresh bedrock to an erosion surface now covered by strata of Late Cretaceous to postglacial age. In many places the profile is overlain by an interval of relatively pure kaolinitic clay produced by the local reworking of the saprolith in Late Cretaceous time. 


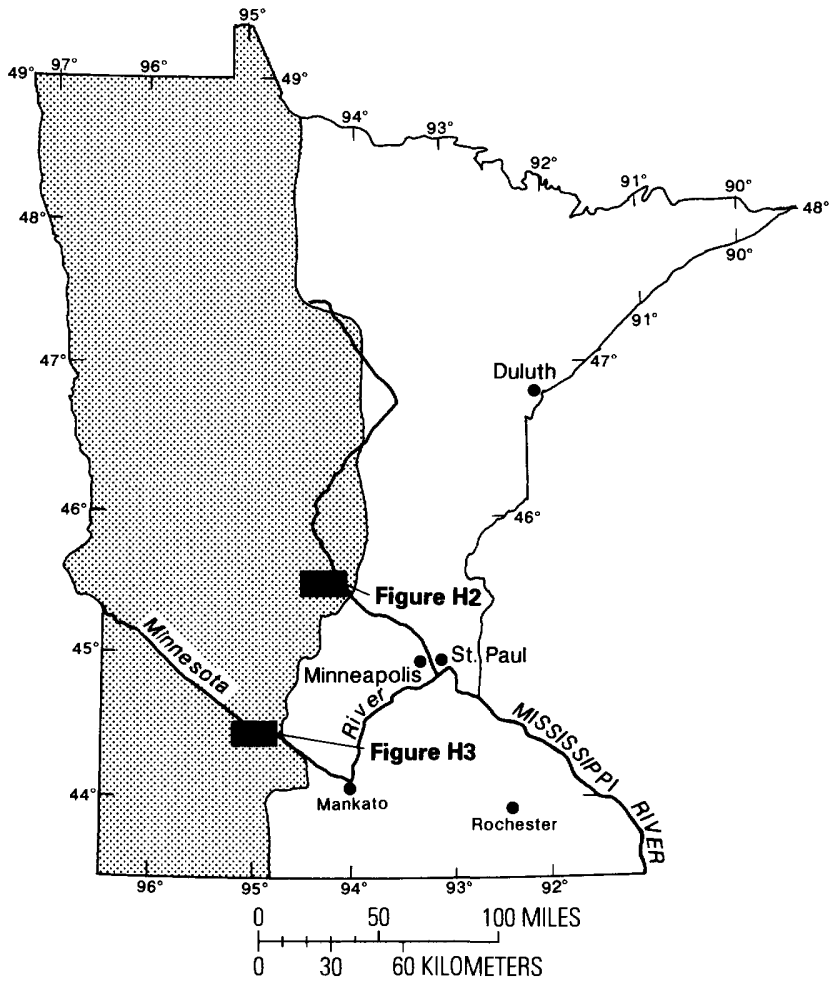

Figure H1. Index map of Minnesota showing the approximate distribution of saprolith and associated weathering products (modified from Parham, 1970). The areas of figures $\mathrm{H}_{2}$ and $\mathrm{H} 3$ are shown as black rectangles.

Detailed mapping, particularly along the Minnesota River Valley in southwestern Minnesota, has shown that the crystalline bedrock in most upland areas has a weathering profile averaging $30 \mathrm{~m}$ in thickness, though profiles are locally as thick as $60 \mathrm{~m}$ (Parham, 1970). Weathered materials are considerably thinner on steep-sided slopes and along valleys cut into the bedrock surface.
Although this kaolinite-rich saprolith was first recognized in outcrops in 1872 (Winchell, 1874), and interest in the commercial development of this resource followed shortly thereafter and cyclically since (Upham, 1888; Hall, 1899; Hall and others, 1911; Grout and Soper, 1919; Bickford and Price, 1947; Parham, 1970; Setterholm and others, 1989; Hauck and others, 1990), very little has been written about the attributes and geologic history of the weathering profile. Much of our knowledge of the processes responsible for its origin was established by S.S. Goldich in a 1936 University of Minnesota doctoral dissertation (Goldich, 1938) that led to the development of the "Goldich stability series."

This report summarizes petrographic, mineralogic, and geochemical properties of the weathering profile as observed at eight localities: three near St. Cloud in east-central Minnesota (figs. $\mathrm{H} 1$ and $\mathrm{H} 2$ ) and five in the Redwood FallsMorgan segment of the Minnesota River Valley in southwestern Minnesota (figs. $\mathrm{H} 1$ and $\mathrm{H} 3$ ). This work was undertaken to provide an understanding of the geologic controls on kaolin occurrence and grade in Minnesota. Others (Prasad and Reid, 1989; Hauck and others, 1990) undertook associated studies of the usefulness of these deposits and means of processing them for industrial use.

\section{METHODOLOGY}

Data for this report were acquired principally to support the possible economic development of kaolinite resources in the State. Therefore, emphasis was given to areas known to be underlain by quartzofeldspathic igneous and metamorphic rocks, particularly where the combined Cretaceous to Quaternary cover was less than $30 \mathrm{~m}$ thick. Actual drilling sites were established from local subsurface

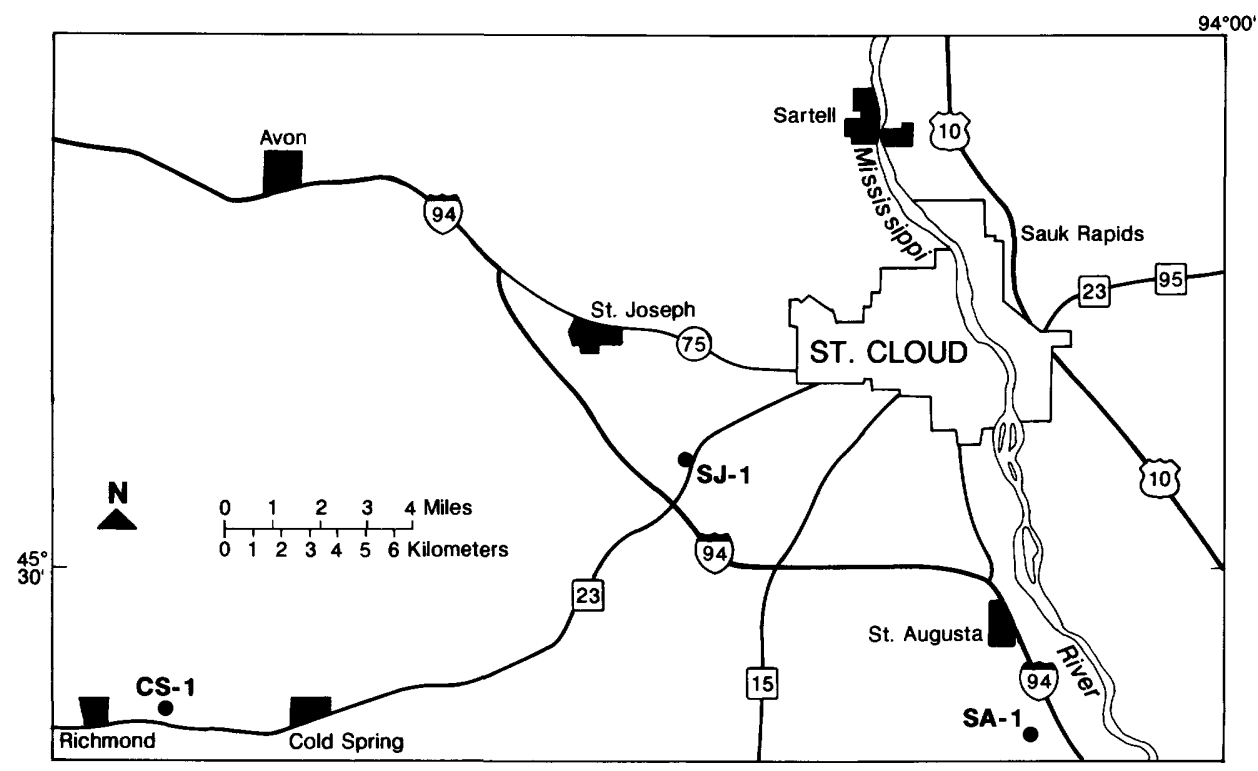

Figure H2. Map of the St. Cloud area, east-central Minnesota, showing the locations of drill holes discussed in the text. CS-1, Cold Spring 1; SA-1, St. Augusta $1 ;$ SJ-1, St. Joseph 1. 


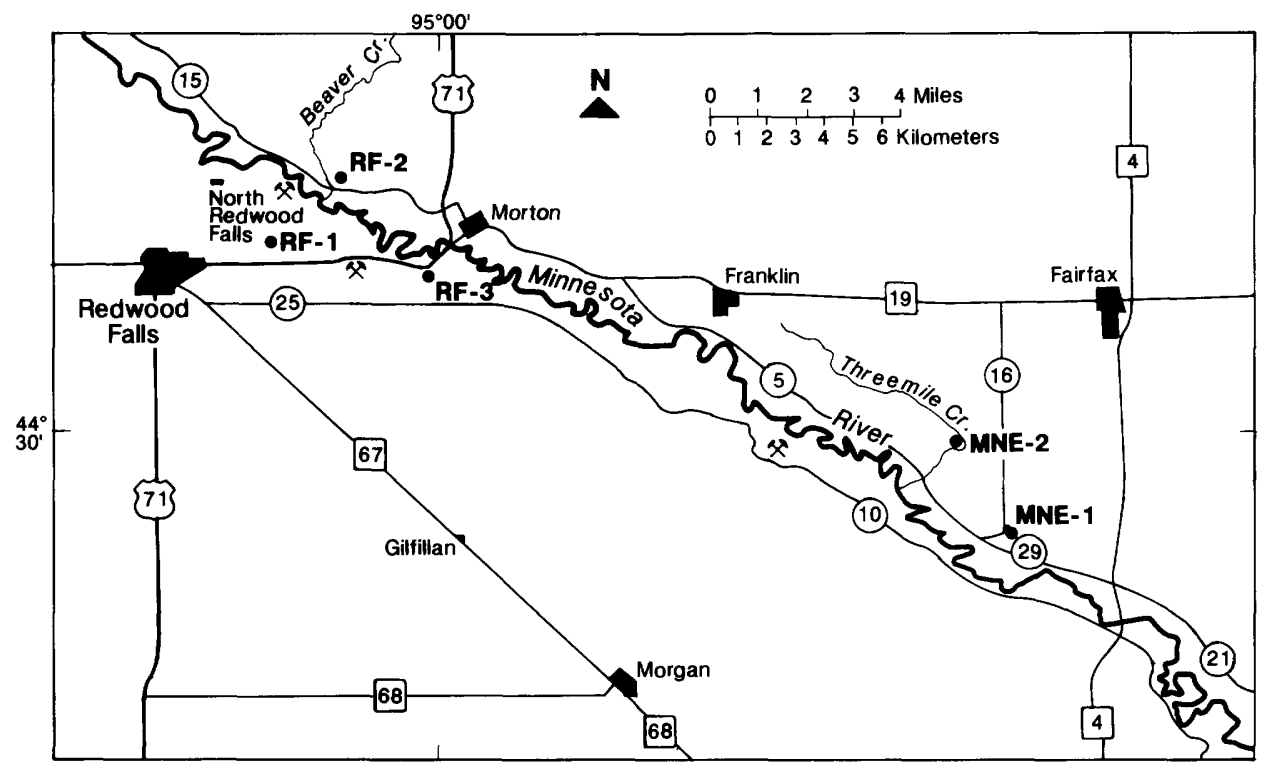

Figure H3. Map of the Redwood Falls-Morgan area along the Minnesota River Valley showing the locations of drill holes discussed in the text. MNE-1, Morgan 1; MNE-2, Morgan 2; RF-1, Redwood Falls 1; RF-2, Redwood Falls 2; RF-3, Redwood Falls 3. Weathering products are mined at the locations indicated by the crossedpick symbol.

information, principally the written $\operatorname{logs}$ of water-well drilling contractors in the files of the Minnesota Geological Survey. Details of the entire site-selection process, as well as the complete data base, are given in Setterholm and others (1989).

In all, $291 \mathrm{~m}$ of weathered residuum and fresh rock were collected and studied. Continuous cores (HX size) having recoveries averaging around 90-95 percent were visually examined in detail, both in the field and again in the laboratory. Thin sections of the parent rocks were prepared and examined by standard microscopy. Descriptions of the cores were used to select samples $12-30 \mathrm{~cm}$ in length for subsequent petrographic, textural, X-ray diffraction, and major and minor element chemical analyses (table H1). Fifty-five X-ray diffraction analyses (example in fig. H4) were performed, including air-dried, glycolated, cation saturated, and heated samples. The clay mineral determinations from this work are qualitative. The relative amount of each mineral was estimated from both the intensity and area enclosed by the diffraction peaks. Because of the framework nature of this study no investigation of the crystallinity of the kaolinite was undertaken. Also, because many samples contained more than one clay type it was not always possible to determine exactly which clay was present at a given site within that core sample. The amount of clay in each core sample was roughly measured by disaggregating it in a sodium pyrophosphate solution and then sieving.

In addition to the analyses listed above, down-hole natural gamma methods were used to further characterize the weathering profile at each test site. The methodology responds principally to the amount of potassium in the profile. Thus, the upper part of an ideal profile-where kaolinite is abundant-is marked by low gamma values; the gamma response typically increases with depth through the saprolith, saprock, weathered rock, and into the parent material. Lateritic clay at the top of the weathering profile is generally a good gamma-emitter because thorium concentrates in it as a lag during weathering (R.S. Lively, oral commun., 1994).

Lastly, as a means of rapidly summarizing the chemical attributes of various parts of the weathering profile, we have relied heavily on the use of the chemical index of alteration (CIA) of Nesbitt and Young (1982). This index, calculated from whole-rock chemical data, measures the ratio of $\mathrm{Al}_{2} \mathrm{O}_{3}$ to $\mathrm{Al}_{2} \mathrm{O}_{3}$ plus $\mathrm{K}_{2} \mathrm{O}$ plus $\mathrm{Na}_{2} \mathrm{O}$ plus $\mathrm{CaO}$. As Goldich (1938) showed, chemical weathering predominantly involves the dissolution of feldspars and the concurrent removal of calcium, sodium, and potassium from the weathering profile by through-flowing solutions. As the rock weathers, alumina is concentrated in the remaining residue and thus is available to form kaolinite. Thus, CIA values increase as weathering progresses. Unaltered granitic rocks typically have CIA values between 45 and 55; kaolinite-rich units have values approaching 100.

\section{MESOZOIC PALEOGEOGRAPHY}

The precise age of the weathering profile and rate at which it was produced remain unresolved. The profile is overlain by strata of Late Cretaceous (Cenomanian to Coniacian) age (Setterholm, 1994), but paleomagnetic data (Tyler and Bailey, 1961; and Symons, 1966) for oxidized and leached materials imply that weathering may have started on the Mesabi iron range in northern Minnesota (Morey, 1983) in the latter part of Late Jurassic time.

From the mid-Jurassic to the early part of the Late Cretaceous, much of what is now western Minnesota was most likely a moderately dissected upland characterized on 
a regional scale by rounded hills separated by interfluves having a total relief of about 40-45 m (Parham, 1970). However, the front that separates weathered from fresh material had, at least locally, a considerably different configuration. For example, the weathering profile in the St. Joseph area (fig. H2) extends to a depth of more than 36 $\mathrm{m}$, but fresh dimension stone is quarried in the same rock type at the land surface less than $0.5 \mathrm{~km}$ away.

The position of the weathering front was controlled in large part by regional hydraulic gradients related to paleotopography and climate. However, the permeability of the weathering profile itself, once it began to develop, had an effect on local hydraulic gradients. Where the bedrock is dominated by granitic rocks, both nearly vertical, orthogonal joints, and nearly horizontal sheeting joints served as the predominant channelways for ground-water movement. Where the bedrock is dominated by gneissic rocks, ground water flow also followed cleavage planes, mineral layering, and planes of schistosity. The anisotropic structure of the parent material caused ground water to
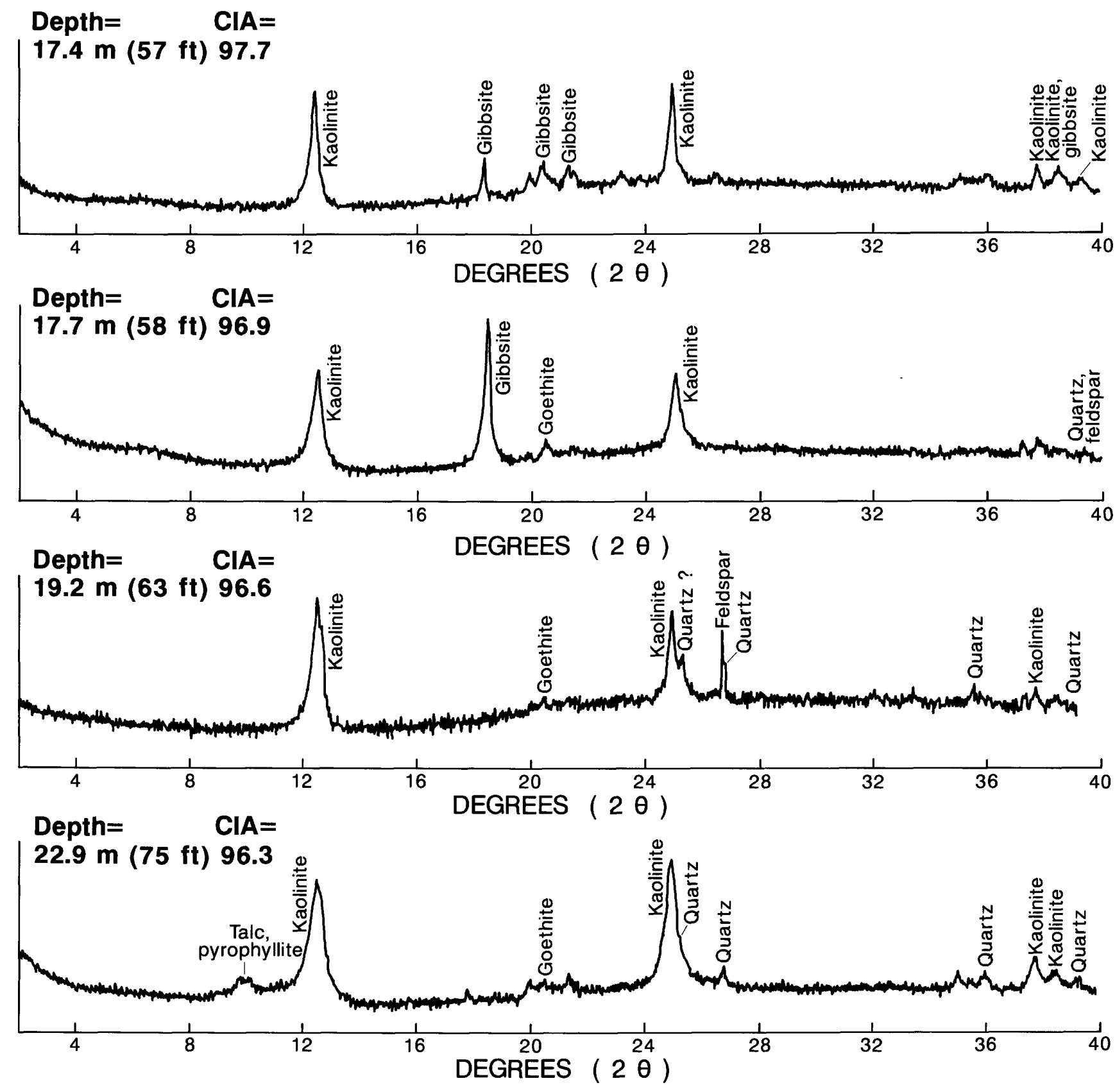

Figure H4 (above and facing page). Representative X-ray diffraction diagrams of the $<2 \mu \mathrm{m}$ clay fraction from the St. Augusta site in east-central Minnesota. Samples air dried. CIA, chemical index of alteration (Nesbitt and Young, 1982). 
flow faster in some directions than in others, and local paleohydraulic gradients rarely can be represented by straight flow paths. The resulting weathering profiles also can be expected to have a marked anisotropic configuration, particularly in the vertical dimension.

Our understanding of climatic conditions during the latter part of Mesozoic time in what is now Minnesota is rather sketchy. In the Late Jurassic, Minnesota was situated generally between lat. $30^{\circ}$ and $40^{\circ} \mathrm{N}$. (Dott and Batten, 1981). The climate most likely was arid to semi-arid as shown by redbeds and associated evaporate deposits in northwestern Minnesota and adjoining parts of North Dakota and Manitoba (Mossler, 1978). These beds were apparently deposited by fluvial processes in channels that were graded to the west and southwest (Moore, 1979). In the Early Cretaceous, Minnesota was located generally $10^{\circ}$ farther north between lat $40^{\circ}$ and $50^{\circ} \mathrm{N}$. in an area affected by seasonal monsoon rains (Dott and Batten, 1981). Parham (1970) suggested that the region was dominated by humid subtropical or tropical conditions. Austin

\section{Depth $=\quad \mathrm{CIA}=$ $29.0 \mathrm{~m} \mathrm{(95} \mathrm{ft)} 75.2$}

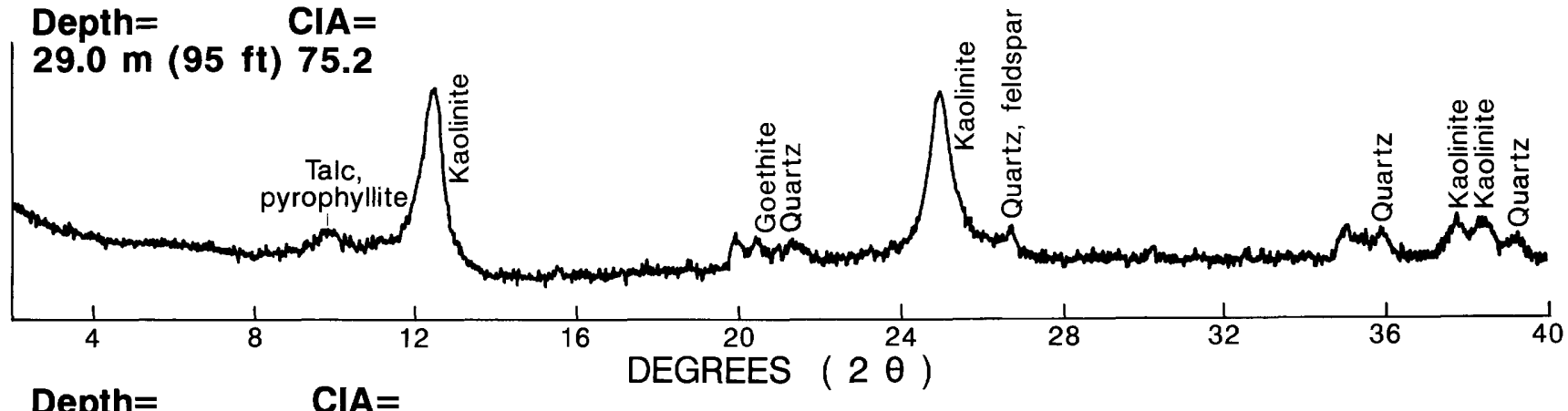

Depth $=\quad \mathrm{CIA}=$

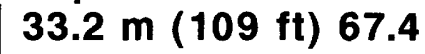
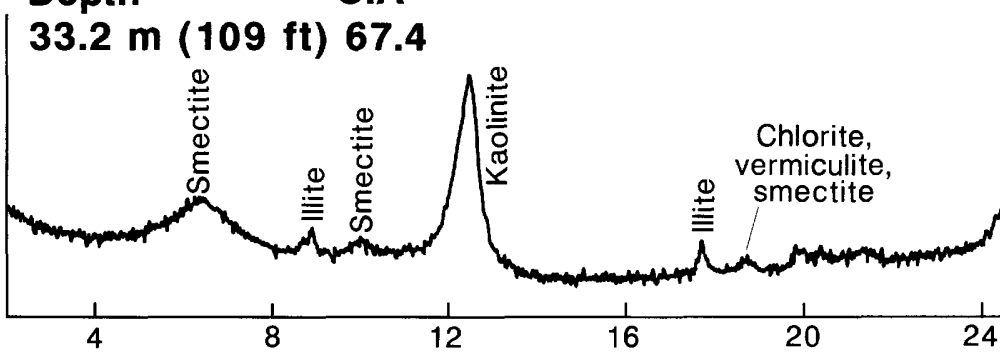

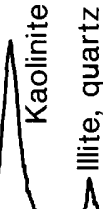

\section{Depth $=$ $\mathrm{CIA}=$ $35.1 \mathrm{~m}$ (115 ft) 47.4}
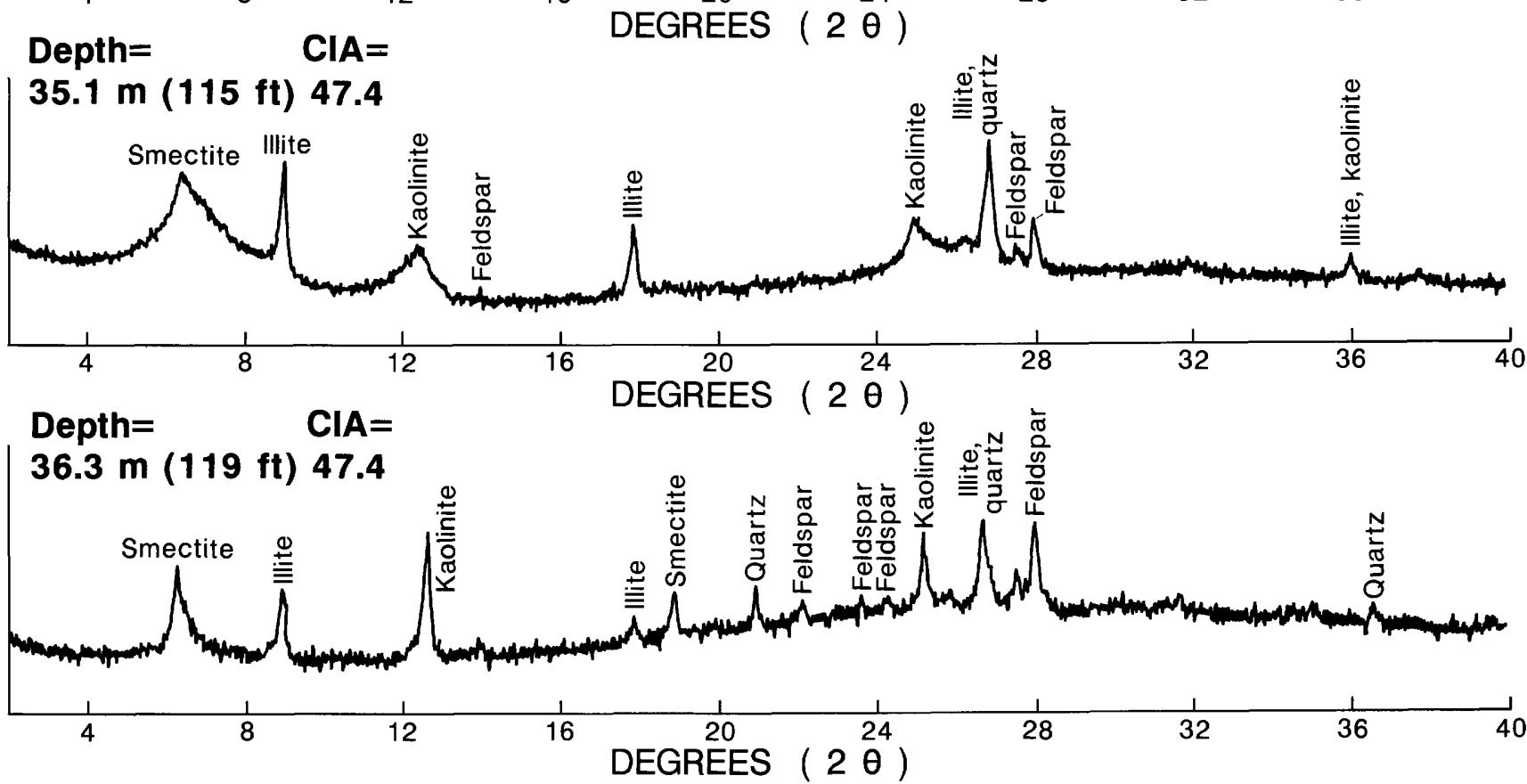

DEGREES $(2 \theta)$

$36.3 \mathrm{~m} \mathrm{(119} \mathrm{ft)} 47.4$ 
(1970) concurred with the idea that humid tropical conditions persisted through early Cenomanian time, but suggested that a significant shift from tropical to temperate climate took place after the weathering profiles had formed and prior to the advance of Late Cretaceous seas into Minnesota in late Cenomanian time. It is this climatic shift, combined with an increase in the height of the water table and the concurrent development of stagnant ground-water conditions, that brought to an end the period of intense chemical weathering in Minnesota.

\section{GENERAL DESCRIPTION OF THE WEATHERING PROFILES}

Approximately $70 \mathrm{~m}$ of saprolith and associated weathering materials were drilled near St. Cloud in eastcentral Minnesota (fig. H2). The profiles at St. Joseph and St. Augusta are developed in granite and quartz monzonite, respectively, whereas the profile at Cold Spring is developed in granodiorite (table H2). The profile at St. Joseph is complicated by alteration products derived from dikes of very coarse grained pegmatite and very fine grained diabasic gabbro. The granodiorite at Cold Spring includes segregations of pegmatitic potassium feldspar and coarse, irregularly shaped inclusions of quartz.

Approximately $184 \mathrm{~m}$ of saprolith and associated weathering products were drilled in the Minnesota River Valley. Two cores totaling $77 \mathrm{~m}$ of material were obtained from two sites north of Morgan and $107 \mathrm{~m}$ from three sites near Redwood Falls (fig. H3). The Archean bedrock that crops out in the valley is a complex migmatitic terrane that varies considerably from place to place. The migmatite is intruded at many places by small to large plutons of generally quartz monzonitic composition (table $\mathrm{H} 3$ ). Thus, the protolith at Morgan 1 is relatively homogeneous and granitic in composition, but the parent material at Morgan 2-only $3.35 \mathrm{~km}$ from Morgan 1 -is a heterogeneous gneiss having amphibolite enclaves interlayered with hornblende-rich and hornblende-poor units of tonalitic composition. The gneiss at Redwood Falls is even more heterogeneous, with at least three compositionally distinct granitic components interlayered on a scale of several centimeters to several meters (table H3). Amphibolite enclaves, biotite-rich schlieren, and biotite-rich pegmatites are scattered throughout the gneiss, as are segregations of coarse-grained potassium feldspar.

The characteristics of each of the weathering profiles are most efficiently conveyed graphically. For each drillhole a composite figure displays depth-based columns showing: (1) the lithologic character of the parent material; (2) the subunits of the weathering profile; (3) the location, number, and CIA value of samples; (4) the gamma log; (5) the
Table H1. Chemical analysis of saprolith and associated weathering products.

[Results in weight percent; column numbers correspond to list at end of table showing drill hole, sample locality, and sample type; NA, not analyzed; LOI, loss on ignition; --, below detection limit]

\begin{tabular}{|c|c|c|c|c|c|}
\hline & 1 & 2 & 3 & 4 & 5 \\
\hline$\overline{\mathrm{SiO}_{2}}$ & 61.0 & 66.1 & 66.7 & 97.0 & 62.9 \\
\hline $\mathrm{Al}_{2} \breve{\mathrm{O}}_{3}$ & 25.1 & 21.3 & 21.4 & 0.74 & 21.3 \\
\hline $\mathrm{CaO}$ & 0.22 & 0.22 & 0.23 & 0.20 & 0.27 \\
\hline $\mathrm{MgO}$ & 0.16 & 0.17 & 0.17 & 0.04 & 0.26 \\
\hline $\mathrm{Na}_{2} \mathrm{O}$ & -- & 0.02 & 0.06 & 0.02 & 0.04 \\
\hline $\mathrm{K}_{2} \mathrm{O}$ & 0.13 & 0.1 & 0.13 & 0.03 & 0.08 \\
\hline \multicolumn{6}{|l|}{ Total } \\
\hline \multicolumn{6}{|l|}{$\mathrm{Fe}$ as } \\
\hline $\mathrm{Fe}_{2} \mathrm{O}_{3}$ & 2.43 & 3.1 & 3.06 & 0.32 & 4.70 \\
\hline $\mathrm{FeO}$ & 1.4 & 1.6 & 1.9 & 0.1 & 3.0 \\
\hline $\mathrm{Fe}_{2} \mathrm{O}_{3}$ & 0.87 & 1.32 & 0.95 & 0.21 & 1.37 \\
\hline $\mathrm{TiO}_{2}$ & 0.57 & 0.62 & 0.48 & 0.08 & 0.55 \\
\hline $\mathrm{MnO}$ & -- & -- & -- & -- & 0.12 \\
\hline $\mathrm{P}_{2} \mathrm{O}_{5}$ & 0.04 & 0.02 & 0.05 & 0.02 & 0.06 \\
\hline LOI & 9.70 & 8.31 & 8.00 & 0.85 & 9.31 \\
\hline $\mathrm{H}_{2} \mathrm{O}^{+}$ & 8.9 & 7.7 & 7.6 & NA & 7.7 \\
\hline $\mathrm{H}_{2}^{2} \mathrm{O}^{-}$ & 0.3 & 0.3 & 0.3 & NA & 0.3 \\
\hline \multirow[t]{2}{*}{$\mathrm{CO}_{2}$} & 0.50 & 0.11 & 0.14 & NA & 1.41 \\
\hline & 6 & 7 & 8 & 9 & 10 \\
\hline $\mathrm{SiO}_{2}$ & 68.1 & 70.0 & 68.8 & 67.9 & 64.3 \\
\hline $\mathrm{Al}_{2} \mathrm{O}_{3}$ & 20.5 & 18.7 & 17.3 & 16.9 & 21.8 \\
\hline $\mathrm{CaO}$ & 0.24 & 0.21 & 0.24 & 0.68 & 0.20 \\
\hline $\mathrm{MgO}$ & 0.20 & 0.44 & 0.53 & 0.90 & 0.20 \\
\hline $\mathrm{Na}_{2} \mathrm{O}$ & 0.02 & 0.03 & 0.09 & 2.45 & 0.03 \\
\hline $\mathrm{K}_{2} \mathrm{O}$ & 0.22 & 1.43 & 2.82 & 3.38 & 0.26 \\
\hline \multicolumn{6}{|l|}{ Total } \\
\hline \multicolumn{6}{|l|}{$\mathrm{Fe}$ as } \\
\hline $\mathrm{Fe}_{2} \mathrm{O}_{3}$ & 2.60 & 2.23 & 3.39 & 3.45 & 3.36 \\
\hline $\mathrm{FeO}$ & 1.3 & 1.1 & 1.8 & 1.5 & 1.8 \\
\hline $\mathrm{Fe}_{2} \mathrm{O}_{3}$ & 1.16 & 1.01 & 1.39 & 1.78 & 1.36 \\
\hline $\mathrm{TiO}_{2}$ & 0.35 & 0.31 & 0.41 & 0.50 & 0.57 \\
\hline $\mathrm{MnO}$ & -- & -- & 0.09 & -- & -- \\
\hline $\mathrm{P}_{2} \mathrm{O}_{5}$ & 0.03 & 0.03 & 0.05 & 0.03 & 0.02 \\
\hline LOI & 7.77 & 6.47 & 5.93 & 3.77 & 8.39 \\
\hline $\mathrm{H}_{2} \mathrm{O}^{+}$ & 7.2 & 6.1 & 5 & 3.5 & 7.9 \\
\hline $\mathrm{H}_{2} \mathrm{O}^{-}$ & 0.3 & 0.2 & 0.2 & 0.5 & -- \\
\hline \multirow[t]{2}{*}{$\mathrm{CO}_{2}$} & 0.35 & 0.38 & 0.67 & 0.02 & 0.40 \\
\hline & 11 & 12 & 13 & 14 & 15 \\
\hline $\mathrm{SiO}_{2}$ & 65.1 & 65.8 & 69.7 & 74.1 & 74.6 \\
\hline $\mathrm{Al}_{2} \mathrm{O}_{3}$ & 18.4 & 18.9 & 15.6 & 13.9 & 13.7 \\
\hline $\mathrm{CaO}$ & 0.16 & 0.14 & 0.16 & 0.09 & 1.22 \\
\hline $\mathrm{MgO}$ & 0.61 & 0.83 & 0.48 & 0.17 & 0.46 \\
\hline $\mathrm{Na}_{2} \mathrm{O}$ & 0.09 & 0.07 & 0.22 & 0.58 & 3.07 \\
\hline $\mathrm{K}_{2} \mathrm{O}$ & 3.20 & 2.27 & 5.44 & 7.34 & 4.48 \\
\hline \multicolumn{6}{|l|}{ Total } \\
\hline \multicolumn{6}{|l|}{$\mathrm{Fe}$ as } \\
\hline $\mathrm{Fe}_{2} \mathrm{O}_{3}$ & 3.95 & 4.33 & 2.92 & 0.71 & 1.28 \\
\hline $\mathrm{FeO}$ & 2.4 & 2.8 & 2.1 & 0.1 & 0.4 \\
\hline $\mathrm{Fe}_{2} \mathrm{O}_{3}$ & 1.28 & 1.22 & 0.59 & 0.60 & 0.84 \\
\hline $\mathrm{TiO}_{2}$ & 0.60 & 0.49 & 0.26 & 0.12 & 0.21 \\
\hline $\mathrm{MnO}$ & -- & -- & -- & -- & -- \\
\hline $\mathrm{P}_{2} \mathrm{O}_{5}$ & 0.04 & 0.03 & 0.02 & 0.02 & 0.07 \\
\hline LOI & 6.85 & 6.77 & 4.54 & 2.54 & 0.93 \\
\hline $\mathrm{H}_{2} \mathrm{O}^{+}$ & 5.7 & 6.4 & 3.6 & 2.1 & 0.9 \\
\hline $\mathrm{H}_{2}^{-} \mathrm{O}^{-}$ & -- & -- & -- & - & -- \\
\hline $\mathrm{CO}_{2}$ & 0.87 & 0.67 & 0.89 & 0.1 & 0.03 \\
\hline
\end{tabular}


Table H1. Chemical analysis of saprolith and associated weathering products - Continued.

\begin{tabular}{|c|c|c|c|c|c|}
\hline & 16 & 17 & 18 & 19 & 20 \\
\hline $\mathrm{SiO}_{2}$ & 71.0 & 72.4 & 73.5 & 55.1 & 66.0 \\
\hline $\mathrm{Al}_{2} \mathrm{O}_{3}$ & 15.0 & 15.0 & 14.0 & 24.4 & 19.7 \\
\hline $\mathrm{CaO}$ & 0.99 & 0.75 & 1.43 & 0.20 & 0.20 \\
\hline $\mathrm{MgO}$ & 0.71 & 0.53 & 0.34 & 0.27 & 0.20 \\
\hline $\mathrm{Na}_{2} \mathrm{O}$ & 2.41 & 3.32 & 3.67 & 0.19 & 0.37 \\
\hline $\mathrm{K}_{2} \mathrm{O}$ & 3.59 & 4.79 & 4.39 & 0.09 & 0.10 \\
\hline \multicolumn{6}{|l|}{$\begin{array}{l}\text { Total } \\
\text { Fe as }\end{array}$} \\
\hline $\begin{array}{l}\mathrm{Fe} \text { as } \\
\mathrm{Fe}_{2} \mathrm{O}\end{array}$ & 248 & 133 & 105 & 650 & 370 \\
\hline $\mathrm{FeO}$ & $\begin{array}{l}2.48 \\
1.4\end{array}$ & $\begin{array}{l}1.33 \\
0.4\end{array}$ & $\begin{array}{l}1.03 \\
0.4\end{array}$ & $\begin{array}{l}0.54 \\
5.3\end{array}$ & $\begin{array}{l}3.10 \\
3.3\end{array}$ \\
\hline $\mathrm{Fe}_{2} \mathrm{O}_{3}$ & 0.92 & 0.89 & 0.61 & 0.70 & 0.03 \\
\hline $\mathrm{TiO}_{2}$ & 0.15 & 0.08 & 0.10 & 0.47 & 0.43 \\
\hline $\mathrm{MnO}$ & 0.12 & -- & -- & -- & -- \\
\hline $\mathrm{P}_{2} \mathrm{O}_{5}$ & 0.03 & 0.02 & 0.04 & 0.02 & 0.04 \\
\hline LOI & 3.08 & 1.85 & 0.93 & 12.0 & 8.70 \\
\hline $\mathrm{H}_{2} \mathrm{O}^{+}$ & 2.6 & 1.0 & 0.3 & 8.8 & 7.2 \\
\hline $\mathrm{H}_{2} \mathrm{O}^{-}$ & -- & 0.2 & 0.1 & -- & -- \\
\hline \multirow[t]{2}{*}{$\mathrm{CO}_{2}$} & 0.60 & 0.09 & 0.08 & 3.47 & 1.64 \\
\hline & 21 & 22 & 23 & 24 & 25 \\
\hline $\mathrm{SiO}_{2}$ & 63.5 & 69.2 & 70.3 & 67.6 & 67.7 \\
\hline $\mathrm{Al}_{2} \mathrm{O}_{3}$ & 21.1 & 20.2 & 20.6 & 21.6 & 21.4 \\
\hline $\mathrm{CaO}$ & 0.20 & 0.12 & 0.13 & 0.12 & 0.12 \\
\hline $\mathrm{MgO}$ & 0.22 & 0.10 & 0.07 & 0.10 & 0.11 \\
\hline $\mathrm{Na}_{2} \mathrm{O}$ & 0.30 & 0.02 & 0.03 & 0.03 & 0.05 \\
\hline $\mathrm{K}_{2} \mathrm{O}$ & 0.23 & 0.08 & 0.08 & 0.06 & 0.11 \\
\hline \multicolumn{6}{|l|}{ Total } \\
\hline \multicolumn{6}{|l|}{$\mathrm{Fe}$ as } \\
\hline $\mathrm{Fe}_{2} \mathrm{O}_{3}$ & 3.79 & 1.96 & 0.77 & 1.47 & 1.49 \\
\hline $\mathrm{FeO}$ & 3.0 & 0.8 & 0.2 & 0.6 & 0.4 \\
\hline $\mathrm{Fe}_{2} \mathrm{O}_{3}$ & 0.46 & 1.07 & 0.55 & 0.8 & 1.05 \\
\hline $\mathrm{TiO}_{2}$ & 0.42 & 0.35 & 0.21 & 0.24 & 0.21 \\
\hline $\mathrm{MnO}$ & -- & - & -- & - & -- \\
\hline $\mathrm{P}_{2} \mathrm{O}_{5}$ & 0.05 & 0.02 & 0.02 & 0.02 & 0.03 \\
\hline LOI & 9.16 & 7.93 & 7.77 & 8.16 & 8.31 \\
\hline $\mathrm{H}_{2} \mathrm{O}^{+}$ & 7.6 & 7.3 & 7.4 & 7.8 & 7.7 \\
\hline $\mathrm{H}_{2}^{2} \mathrm{O}^{-}$ & -- & -- & -- & -- & -- \\
\hline \multirow[t]{2}{*}{$\mathrm{CO}_{2}$} & 1.52 & 0.29 & 0.1 & 0.54 & 0.19 \\
\hline & 26 & 27 & 28 & 29 & 30 \\
\hline $\mathrm{SiO}_{2}$ & 67.7 & 71.5 & 69.5 & 72.4 & 73.5 \\
\hline $\mathrm{Al}_{2} \mathrm{O}_{3}$ & 19.6 & 17.8 & 14.5 & 15.0 & 13.7 \\
\hline $\mathrm{CaO}$ & 0.20 & 0.11 & 0.28 & 0.51 & 0.52 \\
\hline $\mathrm{MgO}$ & 0.27 & 0.13 & 0.46 & 0.33 & 0.39 \\
\hline $\mathrm{Na}_{2} \mathrm{O}$ & -. & 0.10 & 0.99 & 3.16 & 3.68 \\
\hline $\mathrm{K}_{2} \mathrm{O}$ & 0.57 & 3.95 & 5.90 & 5.50 & 4.77 \\
\hline \multicolumn{6}{|l|}{ Total } \\
\hline $\mathrm{Fe}$ as & & & & & \\
\hline $\mathrm{Fe}_{2} \mathrm{O}_{3}$ & 2.91 & 0.67 & 3.39 & 1.54 & 1.78 \\
\hline $\mathrm{FeO}$ & 1.8 & 0.2 & 2.0 & 0.5 & 0.6 \\
\hline $\mathrm{Fe}_{2} \mathrm{O}_{3}$ & 0.91 & 0.45 & 1.17 & 0.98 & 1.11 \\
\hline $\mathrm{TiO}_{2}$ & 0.19 & 0.14 & 0.22 & 0.24 & 0.35 \\
\hline $\mathrm{MnO}$ & -- & -- & -- & -- & -- \\
\hline $\mathrm{P}_{2} \mathrm{O}_{5}$ & 0.03 & 0.02 & 0.07 & 0.08 & 0.10 \\
\hline LOl & 7.62 & 4.85 & 3.77 & 1.54 & 0.93 \\
\hline $\mathrm{H}_{2} \mathrm{O}^{+}$ & 6.9 & 4.7 & 2.4 & 1.3 & 1.0 \\
\hline $\mathrm{H}_{2} \mathrm{O}^{-}$ & -- & -- & -- & - & -- \\
\hline $\mathrm{CO}_{2}$ & 0.98 & 0.06 & 1.26 & 0.32 & 0.06 \\
\hline
\end{tabular}

Table H1. Chemical analysis of saprolith and associated weathering products-Continued.

\begin{tabular}{|c|c|c|c|c|c|}
\hline & 31 & 32 & 33 & 34 & 35 \\
\hline $\mathrm{SiO}_{2}$ & 51.2 & 68.6 & 59.1 & 61.0 & 62.3 \\
\hline $\mathrm{Al}_{2} \mathrm{O}_{3}$ & 23.1 & 15.7 & 18.6 & 15.9 & 16.3 \\
\hline $\mathrm{CaO}$ & 0.38 & 0.26 & 0.41 & 1.16 & 1.98 \\
\hline $\mathrm{MgO}$ & 1.07 & 0.41 & 0.98 & 1.19 & 1.03 \\
\hline $\mathrm{Na}_{2} \mathrm{O}$ & 0.15 & 0.24 & 0.40 & 1.85 & 3.58 \\
\hline $\mathrm{K}_{2} \mathrm{O}$ & 4.09 & 7.12 & 6.56 & 5.10 & 4.20 \\
\hline \multicolumn{6}{|l|}{ Total } \\
\hline \multicolumn{6}{|l|}{$\mathrm{Fe}$ as } \\
\hline $\mathrm{Fe}_{2} \mathrm{O}_{3}$ & 9.16 & 2.74 & 7.44 & 7.64 & 6.29 \\
\hline $\mathrm{FeO}$ & 5.5 & 1.8 & 5.1 & 5.0 & 3.6 \\
\hline $\mathrm{Fe}_{2} \mathrm{O}_{3}$ & 3.05 & 0.74 & 1.77 & 2.08 & 2.29 \\
\hline $\mathrm{TiO}_{2}$ & 1.56 & 0.42 & 0.89 & 0.86 & 1.04 \\
\hline $\mathrm{MnO}$ & 0.15 & -- & 0.10 & 0.08 & -- \\
\hline $\mathrm{P}_{2} \mathrm{O}_{5}$ & 0.08 & 0.03 & 0.05 & 0.2 & 0.38 \\
\hline LOI & 8.93 & 3.31 & 5.77 & 5.23 & 2.39 \\
\hline $\mathrm{H}_{2} \mathrm{O}^{+}$ & 7.3 & 2.8 & 4.2 & 3.2 & 2.2 \\
\hline $\mathrm{H}_{2}^{-} \mathrm{O}^{-}$ & 0.3 & 0.1 & 0.2 & 0.4 & 0.4 \\
\hline \multirow[t]{2}{*}{$\mathrm{CO}_{2}$} & 1.63 & 0.60 & 1.67 & 2.33 & 0.36 \\
\hline & 36 & 37 & 38 & 39 & 40 \\
\hline $\mathrm{SiO}_{2}$ & 37.7 & 48.0 & 49.0 & 42.1 & 48.6 \\
\hline $\mathrm{Al}_{2} \mathrm{O}_{3}$ & 17.0 & 22.6 & 19.6 & 29.5 & 15.7 \\
\hline $\mathrm{CaO}$ & 2.38 & 0.98 & 0.85 & 0.40 & 1.12 \\
\hline $\mathrm{MgO}$ & 1.15 & 0.76 & 1.05 & 0.60 & 1.27 \\
\hline $\mathrm{Na}_{2} \mathrm{O}$ & 0.01 & 0.04 & 0.03 & 0.05 & 0.11 \\
\hline $\mathrm{K}_{2} \mathrm{O}$ & 0.12 & 0.14 & 0.23 & 0.25 & 2.39 \\
\hline \multicolumn{6}{|l|}{ Total } \\
\hline \multicolumn{6}{|l|}{$\mathrm{Fe}$ as } \\
\hline $\mathrm{Fe}_{2} \mathrm{O}_{3}$ & 22.5 & 13.5 & 14.8 & 11.5 & 17.3 \\
\hline $\mathrm{FeO}$ & 18.1 & 9.6 & 11.1 & 5.9 & 13.0 \\
\hline $\mathrm{Fe}_{2} \mathrm{O}_{3}$ & 2.38 & 2.83 & 2.46 & 4.94 & 2.85 \\
\hline $\mathrm{TiO}_{2}$ & 0.74 & 0.81 & 0.80 & 2.07 & 0.69 \\
\hline $\mathrm{MnO}$ & 0.63 & 0.19 & 0.24 & 0.13 & 0.37 \\
\hline $\mathrm{P}_{2} \mathrm{O}_{5}$ & 0.87 & 0.23 & 0.07 & 0.07 & 0.10 \\
\hline LOI & 17.5 & 13.0 & 13.8 & 12.9 & 12.2 \\
\hline $\mathrm{H}_{2} \mathrm{O}^{+}$ & 6.2 & 8.4 & 7.1 & 11.3 & 5.1 \\
\hline $\mathrm{H}_{2} \mathrm{O}^{-}$ & 0.2 & 0.4 & 0.3 & 0.6 & 0.2 \\
\hline \multirow[t]{2}{*}{$\mathrm{CO}_{2}$} & 12.80 & 5.09 & 7.50 & 1.27 & 8.49 \\
\hline & 41 & 42 & 43 & 44 & 45 \\
\hline $\mathrm{SiO}_{2}$ & 56.3 & 63.1 & 65.3 & 66.7 & 55.1 \\
\hline $\mathrm{Al}_{2} \mathrm{O}_{3}$ & 17.3 & 17.3 & 15.7 & 15.7 & 27.9 \\
\hline $\mathrm{CaO}^{3}$ & 0.61 & 1.26 & 1.47 & 1.90 & 0.29 \\
\hline $\mathrm{MgO}$ & 1.07 & 0.73 & 0.68 & 0.69 & 0.18 \\
\hline $\mathrm{Na}_{2} \mathrm{O}$ & 0.10 & 2.40 & 3.04 & 3.71 & 0.04 \\
\hline $\mathrm{K}_{2} \check{\mathrm{O}}$ & 3.81 & 5.77 & 5.24 & 5.25 & 0.05 \\
\hline \multicolumn{6}{|l|}{ Total } \\
\hline \multicolumn{6}{|l|}{$\mathrm{Fe}$ as } \\
\hline $\mathrm{Fe}_{2} \mathrm{O}_{3}$ & 10.4 & 5.12 & 4.75 & 4.21 & 2.41 \\
\hline $\mathrm{FeO}$ & 7.3 & 2.7 & 2.4 & 2.5 & 0.6 \\
\hline $\mathrm{Fe}_{2} \mathrm{O}_{3}$ & 2.29 & 2.12 & 2.08 & 1.43 & 1.74 \\
\hline $\mathrm{TiO}_{2}$ & 0.65 & 0.62 & 0.56 & 0.54 & 0.59 \\
\hline $\mathrm{MnO}$ & 0.17 & -- & -- & -- & -- \\
\hline $\mathrm{P}_{2} \mathrm{O}_{5}$ & 0.07 & 0.16 & 0.15 & 0.15 & 0.03 \\
\hline LOI & 9.08 & 3.23 & 2.39 & 1.08 & 13.0 \\
\hline $\mathrm{H}_{2} \mathrm{O}^{+}$ & 5.0 & 2.7 & 1.9 & 0.9 & 9.5 \\
\hline $\mathrm{H}_{2}^{-} \mathrm{O}^{-}$ & 0.2 & 0.2 & 0.2 & 0.1 & 0.8 \\
\hline $\mathrm{CO}_{2}$ & 4.52 & 0.54 & 0.55 & 0.28 & 0.02 \\
\hline
\end{tabular}


Table H1. Chemical analysis of saprolith and associated weathering products-Continued.

\begin{tabular}{|c|c|c|c|c|c|}
\hline & 46 & 47 & 48 & 49 & 50 \\
\hline $\mathrm{SiO}_{2}$ & 32.1 & 39.6 & 54.0 & 55.5 & 44.5 \\
\hline $\mathrm{Al}_{2} \mathrm{O}_{3}$ & 33.4 & 23.7 & 19.8 & 25.7 & 16.4 \\
\hline $\mathrm{CaO}$ & 0.48 & 0.38 & 0.48 & 0.33 & 1.18 \\
\hline $\mathrm{MgO}$ & 0.42 & 0.50 & 0.38 & 0.39 & 1.47 \\
\hline $\mathrm{Na}_{2} \mathrm{O}$ & 0.07 & 0.07 & 0.02 & -- & 0.02 \\
\hline $\mathrm{K}_{2} \mathrm{O}$ & 0.07 & 0.03 & 0.02 & 0.36 & 1.16 \\
\hline \multirow{2}{*}{\multicolumn{6}{|c|}{$\mathrm{Fe}$ as }} \\
\hline & & & & & \\
\hline $\mathrm{Fe}_{2} \mathrm{O}_{3}$ & 13.8 & 19.7 & 10.9 & 6.13 & 19.0 \\
\hline $\mathrm{FeO}$ & 9.4 & 15.7 & 8.7 & 2.9 & 14.8 \\
\hline $\mathrm{Fe}_{2} \mathrm{O}_{3}$ & 3.35 & 2.25 & 1.23 & 2.91 & 2.55 \\
\hline $\mathrm{TiO}_{2}$ & 1.02 & 1.08 & 0.79 & 1.00 & 0.72 \\
\hline $\mathrm{MnO}$ & 0.15 & 0.12 & 0.15 & - & 0.20 \\
\hline $\mathrm{P}_{2} \mathrm{O}_{5}$ & 0.11 & 0.04 & 0.04 & 0.07 & 0.17 \\
\hline LOI & 18.7 & 14.1 & 12.5 & 10.8 & 14.9 \\
\hline $\mathrm{H}_{2} \mathrm{O}^{+}$ & 11.3 & 8.5 & 7.0 & 8.4 & 5.1 \\
\hline $\mathrm{H}_{2} \mathrm{O}^{-}$ & 1.5 & 0.6 & 0.4 & 0.5 & 0.2 \\
\hline \multirow[t]{2}{*}{$\mathrm{CO}_{2}$} & 5.28 & 5.03 & 5.12 & 0.84 & 10.2 \\
\hline & 51 & 52 & 53 & 54 & 55 \\
\hline $\mathrm{SiO}_{2}$ & 44.2 & 61.7 & 63.0 & 65.4 & 63.0 \\
\hline $\mathrm{Al}_{2} \mathrm{O}_{3}$ & 15.4 & 20.4 & 17.3 & 14.6 & 15.1 \\
\hline $\mathrm{CaO}$ & 1.26 & 0.36 & 0.61 & 3.57 & 3.77 \\
\hline $\mathrm{MgO}$ & 1.55 & 1.07 & 1.44 & 1.14 & 2.17 \\
\hline $\mathrm{Na}_{2} \mathrm{O}$ & 0.05 & 0.15 & 0.85 & 3.3 & 3.57 \\
\hline $\mathrm{K}_{2} \mathrm{O}$ & 2.81 & 5.37 & 5.43 & 3.93 & 3.72 \\
\hline \multicolumn{6}{|l|}{ Total } \\
\hline \multicolumn{6}{|l|}{$\mathrm{Fe}$ as } \\
\hline $\mathrm{Fe}_{2} \mathrm{O}_{3}$ & 19.0 & 4.13 & 4.84 & 3.17 & 4.48 \\
\hline $\mathrm{FeO}$ & NA & 1.9 & 2.3 & 1.5 & 2.7 \\
\hline $\mathrm{Fe}_{2} \mathrm{O}_{3}$ & 19 & 2.02 & 2.28 & 1.5 & 1.48 \\
\hline $\mathrm{TiO}_{2}$ & 0.68 & 0.78 & 0.71 & 0.57 & 0.61 \\
\hline $\mathrm{MnO}$ & 0.72 & -- & -- & -- & 0.09 \\
\hline $\mathrm{P}_{2} \mathrm{O}_{5}$ & 0.33 & 0.22 & 0.21 & 0.23 & 0.24 \\
\hline LOI & 14.2 & 5.93 & 4.85 & 4.16 & 3.23 \\
\hline $\mathrm{H}_{2} \mathrm{O}^{+}$ & NA & 5.0 & 3.6 & 1.1 & 1.1 \\
\hline $\mathrm{H}_{2}^{2} \mathrm{O}^{-}$ & NA & 0.3 & 0.3 & 0.1 & -- \\
\hline $\mathrm{CO}_{2}$ & 10.6 & 0.18 & 0.34 & 2.40 & 1.90 \\
\hline
\end{tabular}

Drill hole, sample locality, and sample type:

1. Redwood Falls 1; $92 \mathrm{ft}$ below collar; saprolith.

2. Redwood Falls $1 ; 110 \mathrm{ft}$ below collar; saprolith.

3. Redwood Falls 1; $121 \mathrm{ft}$ below collar; saprolith.

4. Redwood Falls 1; $122 \mathrm{ft}$ below collar; quartz vein.

5. Redwood Falls $1 ; 140 \mathrm{ft}$ below collar; saprolith.

6. Redwood Falls 1; $153 \mathrm{ft}$ below collar; saprolith.

amount of each gamma emitter; (6) the amount of clay; and (7) the mineralogy of the clay (figs. H5-H12). More details regarding the protoliths are given in tables $\mathrm{H} 2$ and $\mathrm{H} 3$.

The whole-rock chemistry of the profiles is represented in figures H13-H15. In this series of plots, apparent changes in bulk chemistry are plotted against a vertical depthbased scale. Where the drill holes encountered only part of a weathering profile (due to erosion or drilling limitations), we have combined the samples from sites having similar protoliths on a single plot. Because the samples are from more than one drillhole, it is not meaningful to use absolute depth values. The depth of any sample below the current
Table H1. Chemical analysis of saprolith and associated weathering products-Continued.

7. Redwood Falls $1 ; 167 \mathrm{ft}$ below collar; saprolith.

8. Redwood Falls $1 ; 186.5 \mathrm{ft}$ below collar; weathered gneiss.

9. Redwood Falls $1 ; 219 \mathrm{ft}$ below collar; weathered gneiss.

10. Redwood Falls $2 ; 124 \mathrm{ft}$ below collar; saprolith.

11. Redwood Falls $2 ; 151 \mathrm{ft}$ below collar; saprolith.

12. Redwood Falls $2 ; 158 \mathrm{ft}$ below collar; saprolith.

13. Redwood Falls $2 ; 189.5 \mathrm{ft}$ below collar; saprock.

14. Redwood Falls $2 ; 229 \mathrm{ft}$ below collar; granodiorite gneiss.

15. Redwood Falls $2 ; 261 \mathrm{ft}$ below collar; granodiorite gneiss.

16. Redwood Falls 3; $155 \mathrm{ft}$ below collar; saprock.

17. Redwood Falls $3 ; 159.5 \mathrm{ft}$ below collar; saprock.

18. Redwood Falls 3; $192 \mathrm{ft}$ below collar; granodiorite gneiss.

19. Morgan $2 ; 94.5 \mathrm{ft}$ below collar; saprolith

20. Morgan 2; $124 \mathrm{ft}$ below collar; saprolith.

21. Morgan 2; $136 \mathrm{ft}$ below collar; saprolith.

22. Morgan 2; $170 \mathrm{ft}$ below collar; saprolith.

23. Morgan 2; $182.5 \mathrm{ft}$ below collar; saprolith.

24. Morgan 2; $186 \mathrm{ft}$ below collar; saprolith.

25. Morgan 2; $201.5 \mathrm{ft}$ below collar; saprolith.

26. Morgan 2; $213.5 \mathrm{ft}$ below collar; saprolith.

27. Morgan $2 ; 234 \mathrm{ft}$ below collar; saprolith.

28. Morgan 1;214 ft below collar; saprock.

29. Morgan 1; $242 \mathrm{ft}$ below collar; weathered gneiss.

30. Morgan 1; $256 \mathrm{ft}$ below collar; granitic gneiss.

31. Cold Spring 1; $70 \mathrm{ft}$ below collar; saprolith.

32. Cold Spring 1; $79 \mathrm{ft}$ below collar; saprolith.

33. Cold Spring 1; $88.5 \mathrm{ft}$ below collar; saprolith.

34. Cold Spring 1; $106 \mathrm{ft}$ below collar; saprolith.

35. Cold Spring $1 ; 111 \mathrm{ft}$ below collar; weathered granodiorite

36. St. Joseph $1 ; 32 \mathrm{ft}$ below collar; saprolith.

37. St. Joseph $1 ; 41 \mathrm{ft}$ below collar; saprolith.

38. St. Joseph 1; $55 \mathrm{ft}$ below collar; saprolith.

39. St. Joseph 1; $72 \mathrm{ft}$ below collar; saprolith.

40. St. Joseph 1; $73 \mathrm{ft}$ below collar; saprolith.

41. St. Joseph 1; $82 \mathrm{ft}$ below collar; saprolith.

42. St. Joseph 1; $116 \mathrm{ft}$ below collar; saprock.

43. St. Joseph 1: $135 \mathrm{ft}$ below collar; weathered granite.

44. St. Joseph 1; $156 \mathrm{ft}$ below collar; granite..

45. St. Augusta $1 ; 57 \mathrm{ft}$ below collar; bauxite.

46. St. Augusta 1; $58.5 \mathrm{ft}$ below collar; bauxite.

47. St. Augusta 1; $63 \mathrm{ft}$ below collar; saprolith.

48. St. Augusta $1 ; 67 \mathrm{ft}$ below collar; saprolith.

49. St. Augusta $1 ; 75 \mathrm{ft}$ below collar; saprolith.

50. St. Augusta 1; $82 \mathrm{ft}$ below collar; saprolith.

51. St. Augusta 1:87 ft below collar; saprolith.

52. St. Augusta 1;95 ft below collar; saprolith.

23. St. Augusta 1;109 ft below collar; saprolith.

54. St. Augusta 1; $115 \mathrm{ft}$ below collar; weathered quartz monzonite.

55. St. Augusta 1;119 ft below collar; quartz monzonite.

land surface is unimportant because it mostly reflects how much post-weathering erosion and glacial deposition have occurred. The relative spacing of samples within a weathering profile is important and has been maintained in these figures. Figures $\mathrm{H} 16, \mathrm{H} 17$, and $\mathrm{H} 18$ also illustrate chemical changes with depth, but variations in the amount of each constituent have been normalized against changes in alumina content to account for effects of weathering on bulk density.

The mineralogical changes associated with weathering are illustrated in figure $\mathrm{H} 19$, where modal mineralogy is plotted against CIA, and in figure $\mathrm{H} 20$, where the mineralogy of the clay fraction is plotted against CIA. 
Table H2. Nature of protolith at St. Cloud area sites, eastcentral Minnesota (Streckeisen classification, Streckeisen, 1976).

\begin{tabular}{|c|c|}
\hline Site & Description \\
\hline St. Joseph & $\begin{array}{l}\text { Granite, greenish pink, coarse grained, slightly } \\
\text { porphyritic, massive. Microcline, } 46 \% \text {; plagioclase, } \\
37 \% \text {; quartz, } 10 \% \text {; biotite, } 2 \% \text {; calcite, } 2 \% \text {; } \\
\text { hornblende, } 1 \% \text {; chlorite, } 1 \% \text {; clay minerals, } 1 \% \text {; } \\
\text { traces of apatite, opaque oxides, and zircon. }\end{array}$ \\
\hline Cold Spring & $\begin{array}{l}\text { Granodiorite, slightly weathered, dark green and pink. } \\
\text { Plagioclase, } 40 \% \text {; quartz, } 28 \% \text {; microcline, } 12 \% \text {; } \\
\text { chlorite, } 12 \% \text {; biotite, } 6 \% \text {; apatite, } 1 \% \text {; traces of } \\
\text { opaque oxides, zircon, and calcite. }\end{array}$ \\
\hline St. Augusta & $\begin{array}{l}\text { Quartz monzonite, pink and black, equigranular, } \\
\text { medium grained. Plagioclase, } 44 \% \text {; microcline, } \\
40 \% \text {; quartz, } 10 \% \text {; chlorite (after biotite) } 3 \% \text {; biotite, } \\
1 \% \text {; hornblende, } 1 \% \text {; traces of apatite, zircon, } \\
\text { sphene, epidote, and calcite. }\end{array}$ \\
\hline
\end{tabular}

\section{DISCUSSION}

A general stratigraphic model for the pre-Cretaceous weathering profiles in Minnesota can be constructed from the data presented. A general model is possible mainly because the same mineral constituents - quartz, plagioclase, potassium feldspar, hornblende, and biotite-dominate all of the parent materials. However, the construction of a general profile is complicated by the fact that the specific properties of the profile at any given place are not a simple function of depth beneath the geomorphic surface. The profiles are irregularly zoned because of differences in the stabilities of the primary minerals, which are heterogeneously distributed, and because of differences in the duration and intensity of weathering between the bottom and top of the profile, controlled mainly by complex ground-water flow patterns along structurally controlled channelways. Plotting mineralogical changes against CIA values enables better correlations with weathering intensity.

From a physical perspective, the model of a fully developed profile consists of fresh rock, weathered rock, saprock, saprolith, and lateritic clay. The saprolith can in turn be divided into two subunits. The lower subunit, although composed predominantly of clay minerals, retains the original fabric and structure of the parent material. Chemical reactions there most likely took place under isovolumetric conditions. In contrast, the original fabric and structure in the upper subunit are typically disrupted, implying that it was formed under non-isovolumetric conditions by any number of complexly interrelated mechanical and chemical processes. Textural evidence shows that the lateritic clay also formed from interrelated mechanical and non-isovolumetric chemical processes.
Table H3. Nature of protolith at Redwood Falls area sites, southwestern Minnesota (Streckeisen classification, Streckeisen, 1976).

\begin{tabular}{|c|c|}
\hline Site & Description \\
\hline $\begin{array}{c}\text { Redwood } \\
\text { Falls } 1\end{array}$ & $\begin{array}{l}\text { Three major rock types are present. One is a granitic } \\
\text { gneiss, greenish gray and white, medium grained, } \\
\text { composed of potassium feldspar, } 30 \% \text {; quartz, } 25 \% \text {; } \\
\text { hornblende and biotite, } 25 \% \text {; and plagioclase, } 20 \% \text {. } \\
\text { Another is a pegmatitic phase of pink quartz and } \\
\text { orthoclase. The third is an inequigranular hybrid } \\
\text { gneiss containing coarse, pink, orthoclase } \\
\text { porphyroclasts and an increased granitic component. }\end{array}$ \\
\hline $\begin{array}{r}\text { Redwood } \\
\text { Falls } 2\end{array}$ & $\begin{array}{l}\text { Three types of protolith exist. One is a granitic gneiss, } \\
\text { medium grained, greenish gray and white to pink, } \\
\text { composed of potassium feldspar, } 30-35 \% \text {; quartz, } \\
25 \% \text {; plagioclase, } 20 \% \text {; hornblende, } 20 \% \text {; and } \\
\text { biotite, } 5 \% \text {. Another is a well-foliated granodiorite, } \\
\text { medium to coarse grained, and composed of feldspar, } \\
50 \% \text {; biotite, } 40 \% \text {; and quartz, } 10 \% \text {. The third is } \\
\text { segregations of granitic pegmatite composed of } \\
\text { potassium feldspar, } 50 \% \text {; plagioclase, } 25 \% \text {; and } \\
\text { quartz, } 25 \% \text {. }\end{array}$ \\
\hline $\begin{array}{c}\text { Redwood } \\
\text { Falls } 3\end{array}$ & $\begin{array}{l}\text { The complex protolith includes (1) tonalite, well } \\
\text { foliated, pink and green, containing large } \\
\text { porphyroblasts of pink potassium feldspar, and } \\
\text { variable amounts of mafic minerals from } 15 \text { to } 75 \% \text {; } \\
\text { (2) granite, weakly foliated, pink, equigranular, } \\
\text { medium to coarse grained; (3) a mafic phase, dark } \\
\text { greenish-black with fine white mottling and possible } \\
\text { microdiabasic texture; and (4) amphibolitic enclaves, } \\
\text { dark greenish-black and very rich in foliated, altered } \\
\text { biotite. }\end{array}$ \\
\hline Morgan 1 & $\begin{array}{l}\text { Granitic gneiss, coarse-grained, weakly porphyritic. } \\
\text { Microcline, } 39 \% \text {; plagioclase, } 32 \% \text {; quartz, } 27 \% \text {; } \\
\text { biotite, } 2 \% \text {; trace of apatite and zircon. }\end{array}$ \\
\hline Morgan 2 & No protolith recovered. \\
\hline
\end{tabular}

\section{Mineralogical Attributes of the Model}

\section{Slightly Weathered Zone}

The lowest zone in the weathering profile involves slightly weathered rock that is typically $2-30 \mathrm{~cm}$ thick. Mineral alteration is confined to fracture faces and to where the ferrous iron component of mafic minerals has been oxidized to the ferric state to produce iron staining and plagioclase grains have been etched locally along cleavage planes. No clay minerals were detected by X -ray diffraction in this slightly weathered rock.

\section{Saprock}

Saprock is transitional between weathered rock below and saprolith above. Though it contains appreciable clay minerals, saprock is fairly rigid. Rigidity is maintained by grain-to-grain bonds inherited from original magmatic crystallization or subsequent metamorphic 
recrystallization. The lowermost few centimeters of the saprock are characterized by grains of white and chalky plagioclase and by hornblende partly replaced by green clay, mostly a mixture of chlorite and smectite. Other minerals such as quartz, potassium feldspar, and biotite seem unaffected. Toward the top of the saprock, nearly all of the hornblende is altered to chlorite and much of the green clay is a mixture of illite and kaolinite. Plagioclase is replaced by a white clay, which is mostly a mixture of kaolinite and illite, and biotite is replaced by vermiculite. Quartz and potassium feldspar remain relatively unaffected. A textural correlation between plagioclase, hornblende, and associated clays implies that the saprock was formed by isovolumetric weathering processes.

\section{Saprolith}

Much of the saprolith, like the underlying saprock, has textural attributes that mimic those of the protolith. Although there is a transitional interval of $1 \mathrm{~m}$ or so between them, the saprolith is marked by the complete dissolution of hornblende and nearly complete dissolution of plagioclase. Therefore, saprolith is considerably less indurated than saprock. The modal proportions of biotite and potassium feldspar also decrease, whereas kaolinite increases stratigraphically upward in the saprolith. The level where nearly all of the biotite and much of the potassium feldspar disappear generally corresponds to the level where primary igneous or metamorphic texture disappears. Above that level, the saprolith is dominated by kaolinite, a minor amount of quartz, and a trace amount of potassium feldspar.

Ideally, the development of saprolith is dependent upon a mixture of primary minerals that alter at different rates. Different dissolution rates selectively remove the more reactive mineral species-such as the amphiboles and plagioclase. The remaining, more resistant minerals - such as potassium feldspar and quartz-provide the supporting structural framework that is essential to maintain isovolumetric weathering conditions.

In the upper part of the saprolith, however, dissolution of even the most resistant minerals leads to a breakdown in textural integrity. This, in turn, leads to a physical environment where non-isovolumetric chemical processes can prevail. Chemical changes in this zone may reflect absolute losses, but absolute gains also can take place because of the physical translocation of particulate mineral constituents.

The relative instabilities of silicate minerals, as inferred from the generalized weathering profile described above, are very similar to those first documented by Goldich (1938). However, we observed that amphiboles alter first, followed by biotite, and that both dissolve more readily than the feldspars, which dissolve more readily than quartz. Of the feldspars, plagioclase is much more reactive than potassium feldspar. Quartz persists as a discrete primary phase in all but the most intensely weathered saprolith.
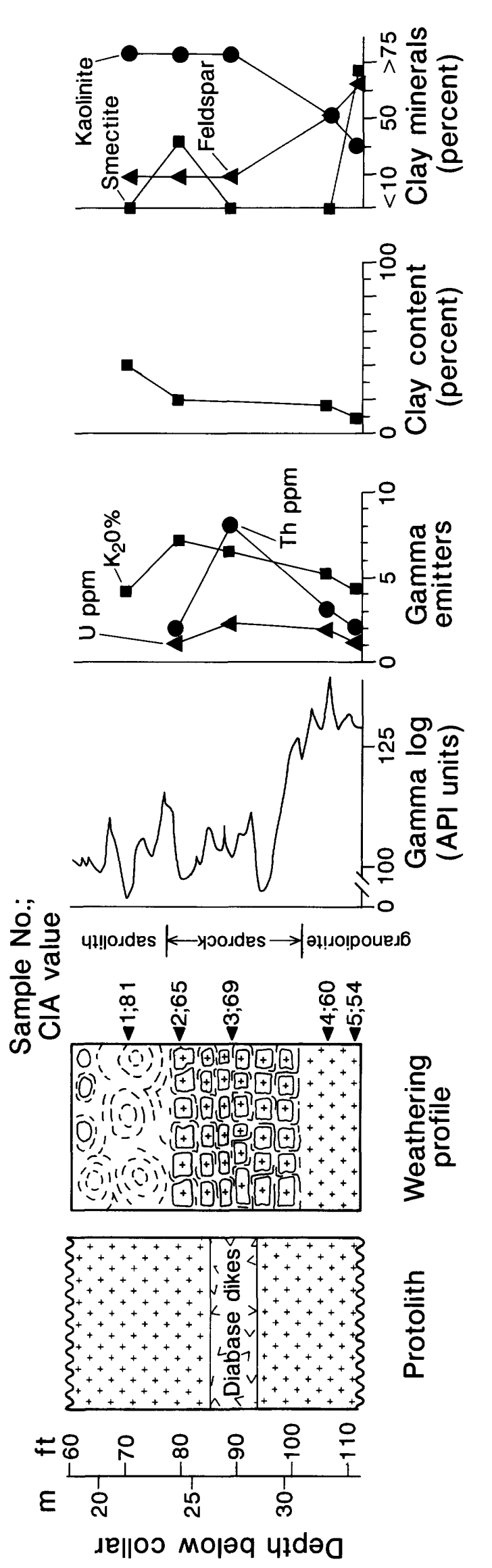

范 


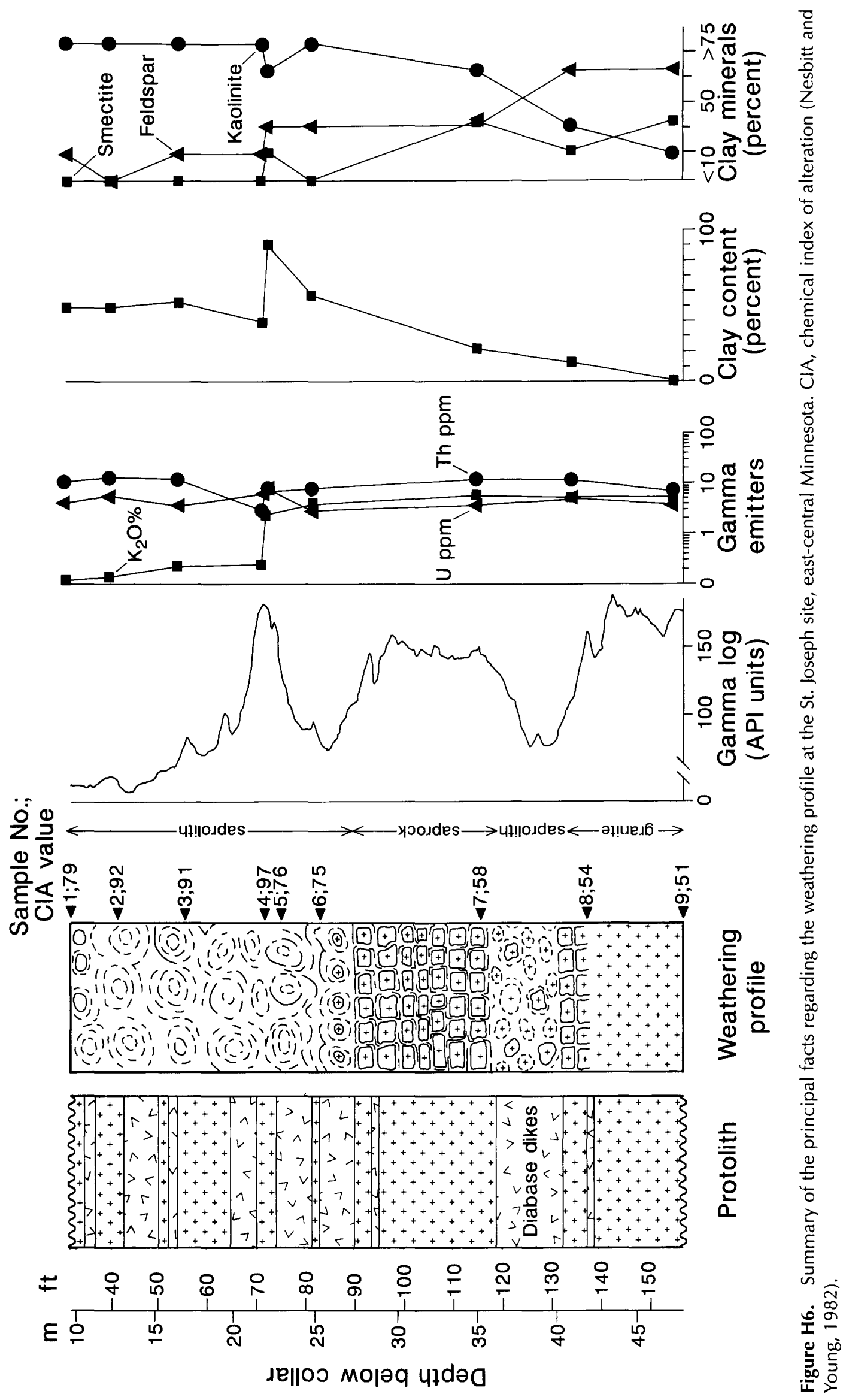




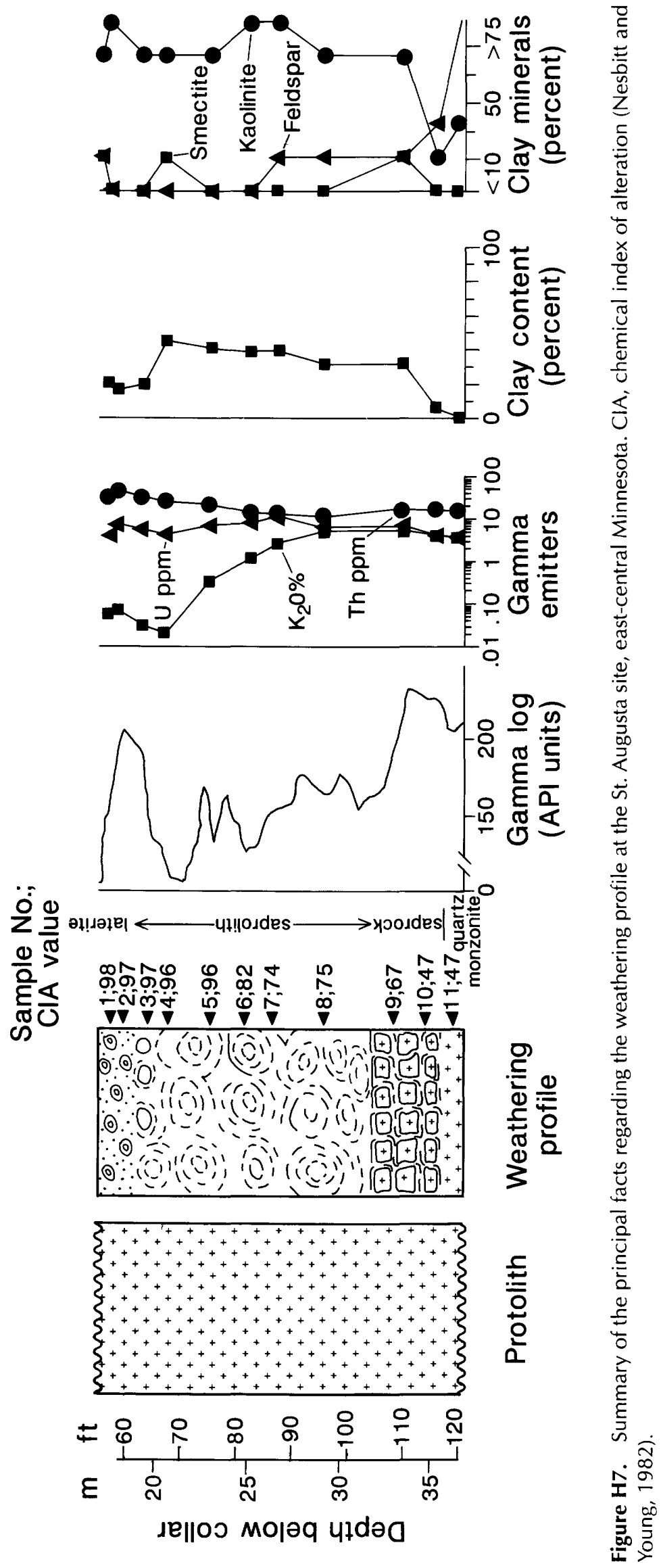




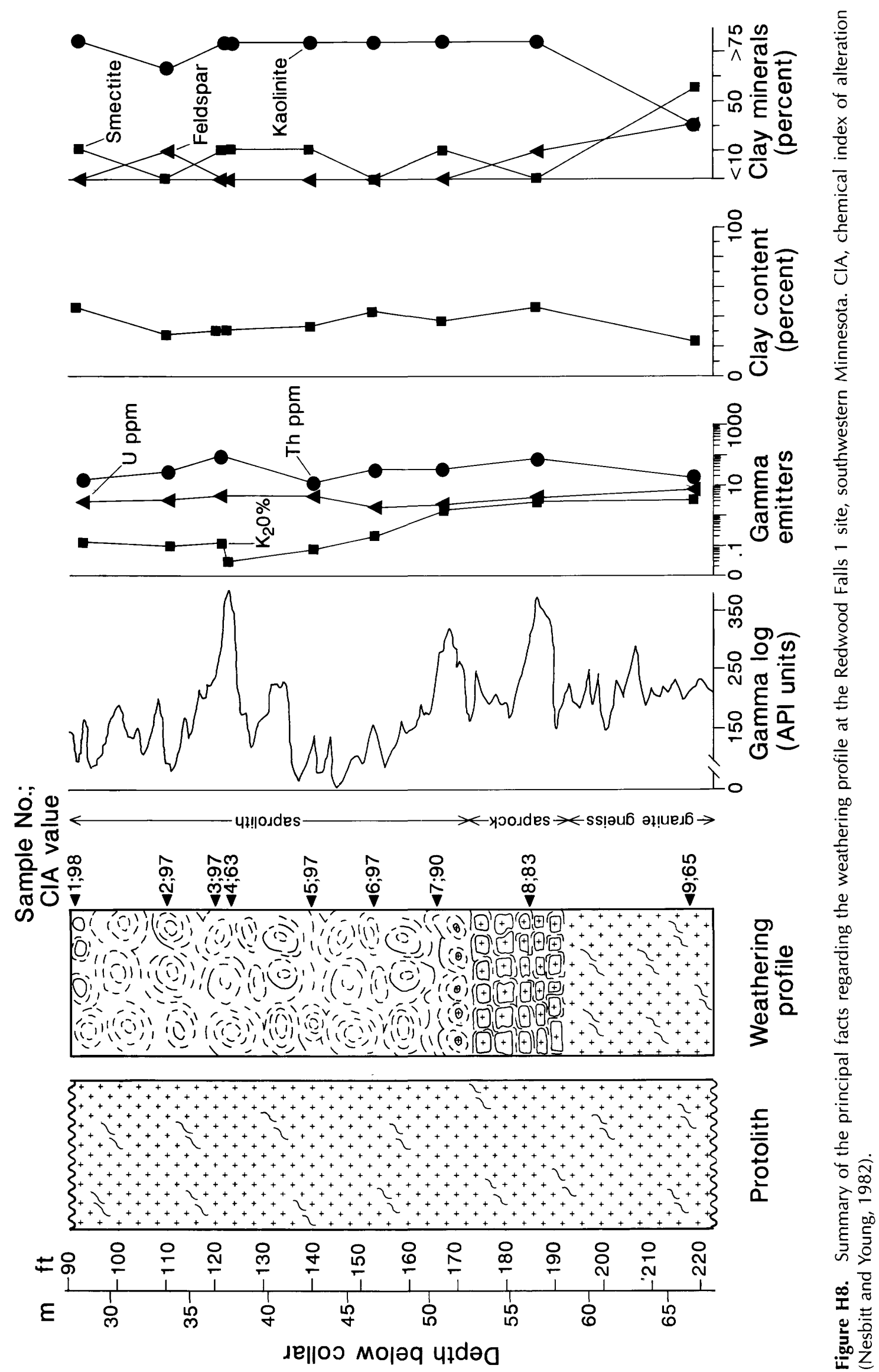




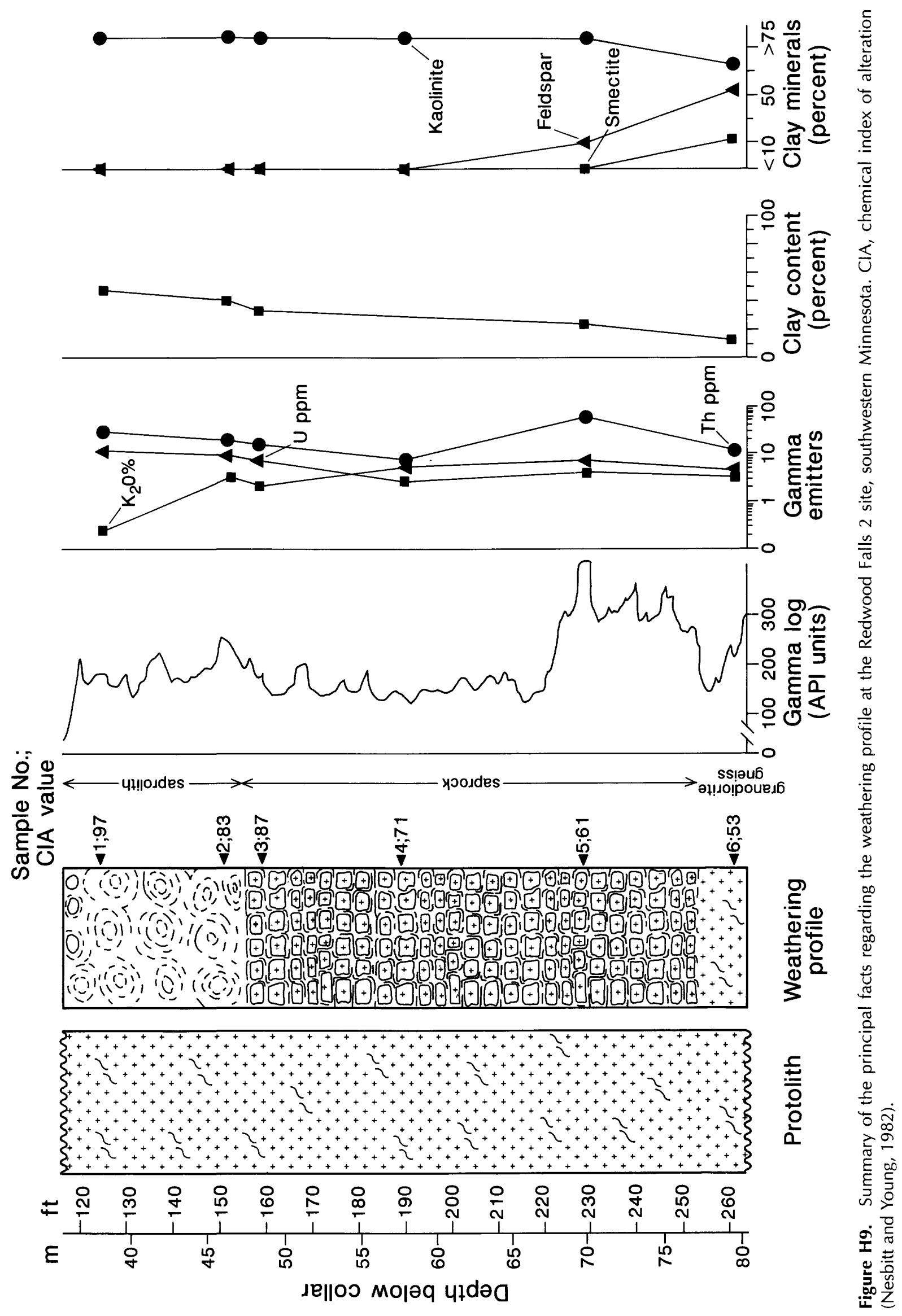




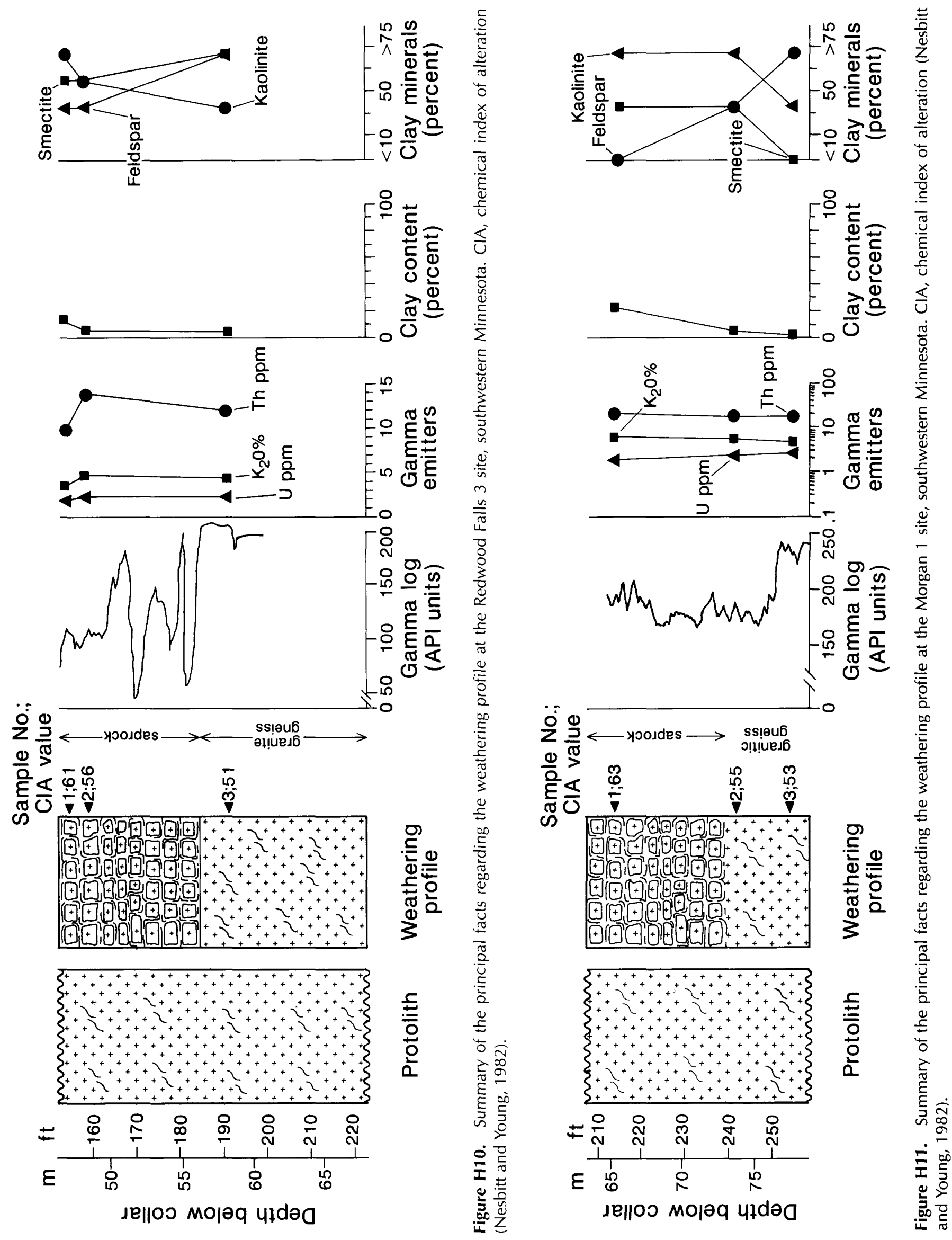




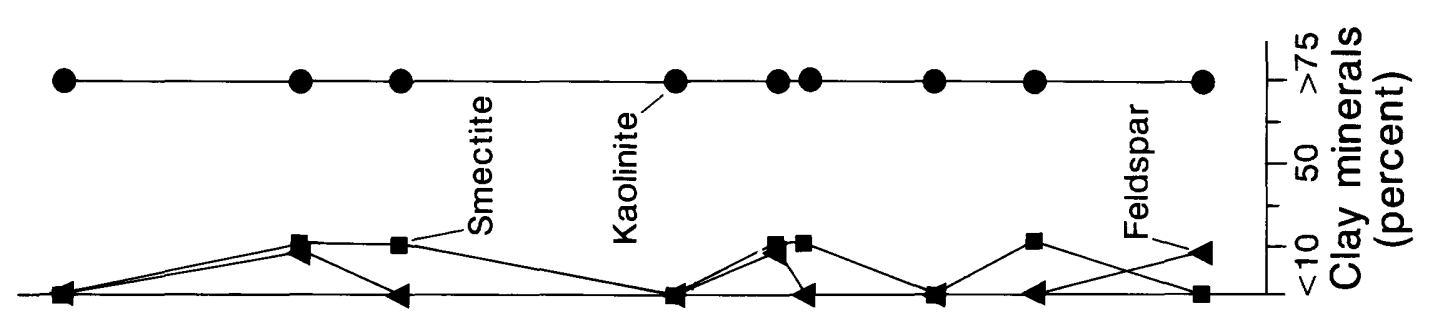

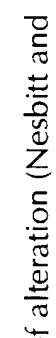
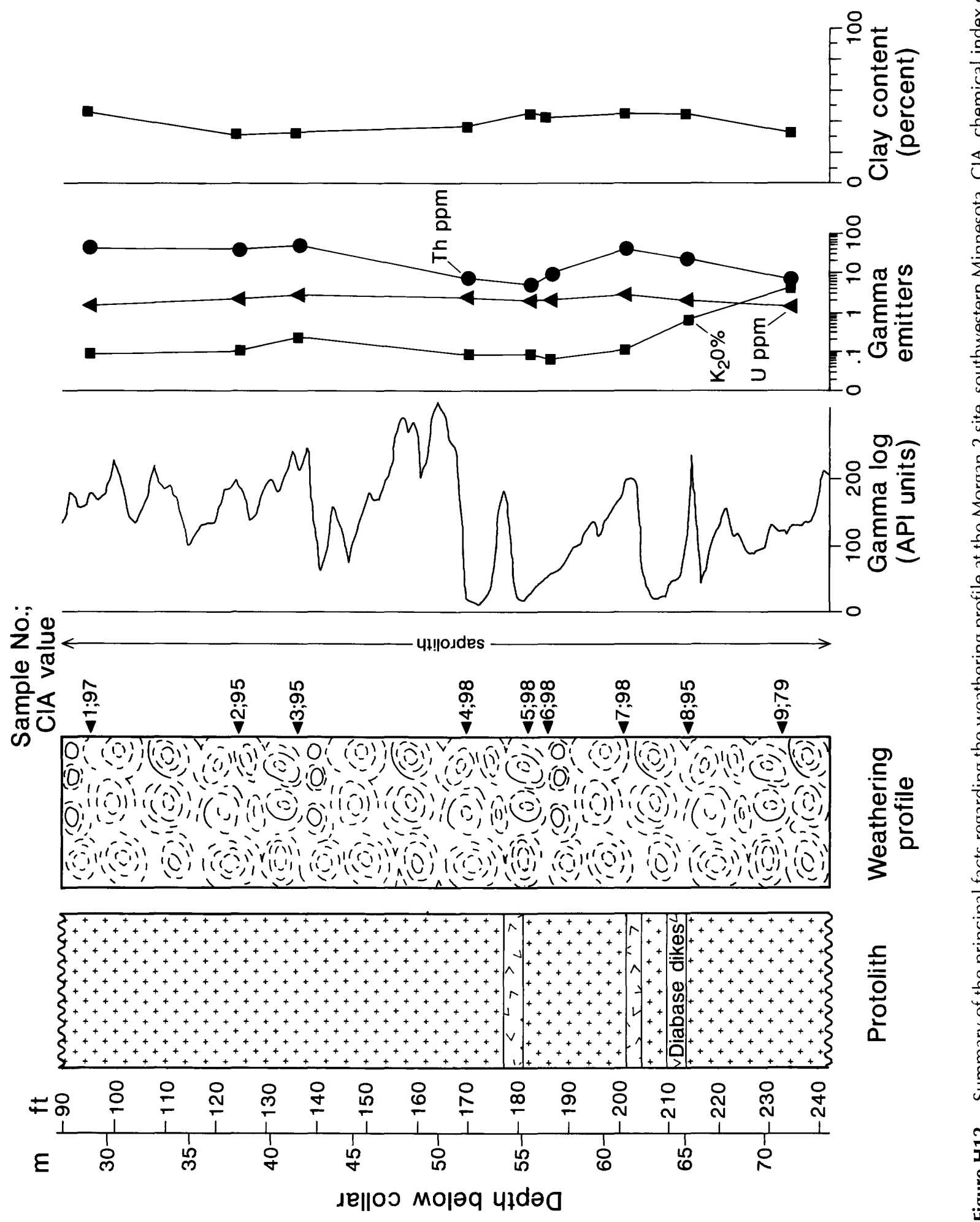

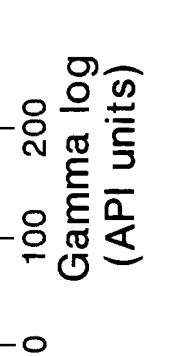
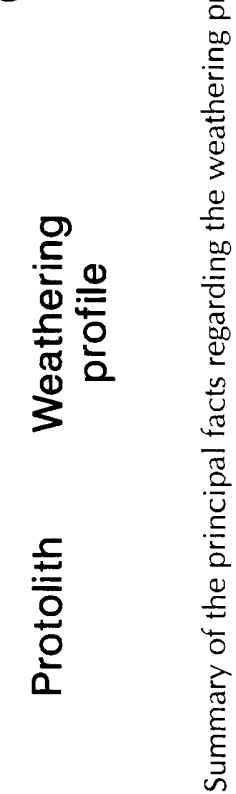

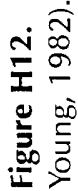



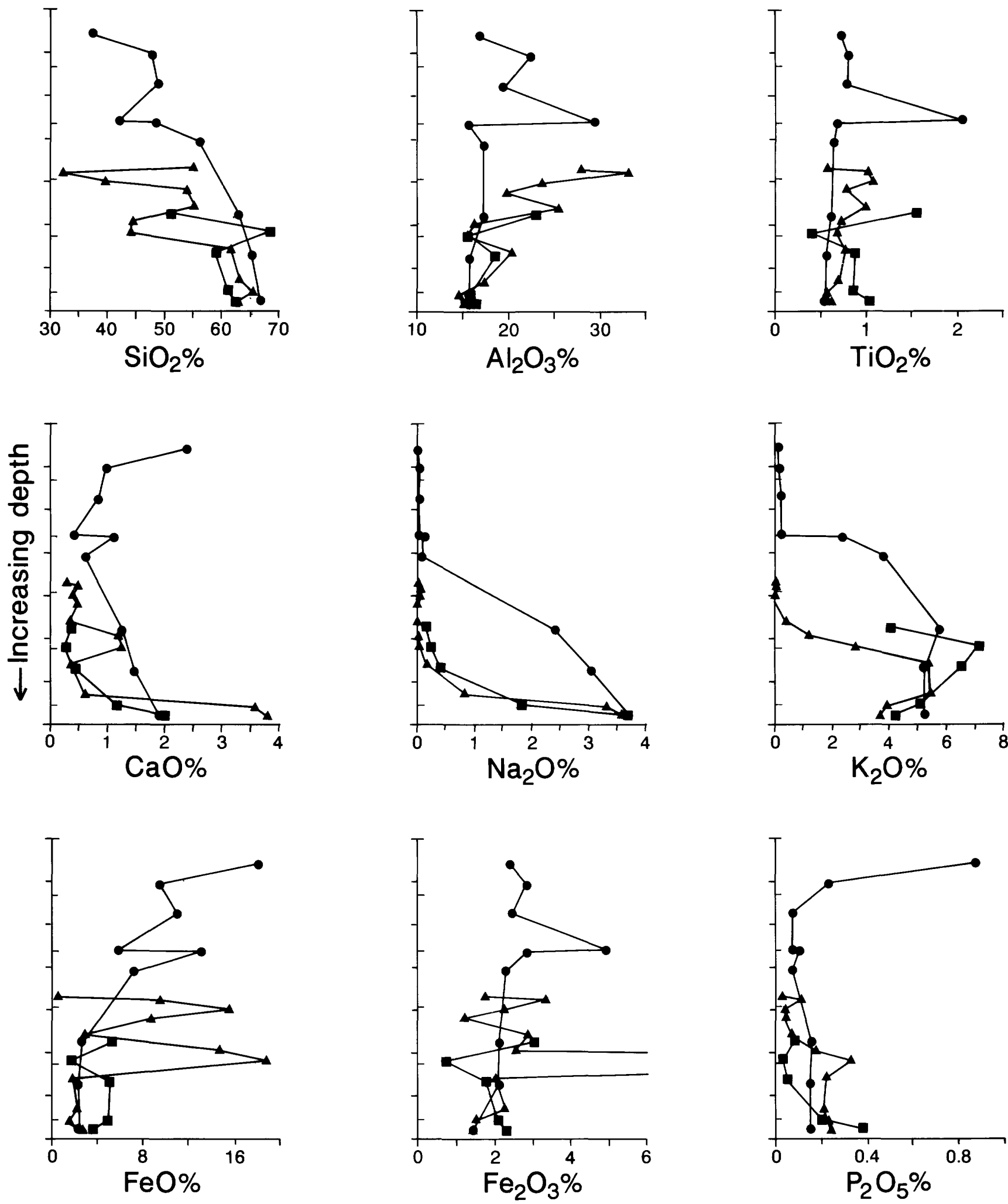

Figure H13. Whole-rock chemical trends at the St. Cloud area sites, east-central Minnesota. The data from all three sites are arranged on a single plot and positioned according to the degree of weathering in each profile. The outside ticks on the vertical axis are 50-ft increments, and the inside ticks are 20-m increments. Dot, St. Joseph; triangle, St. Augusta; square, Cold Spring. 

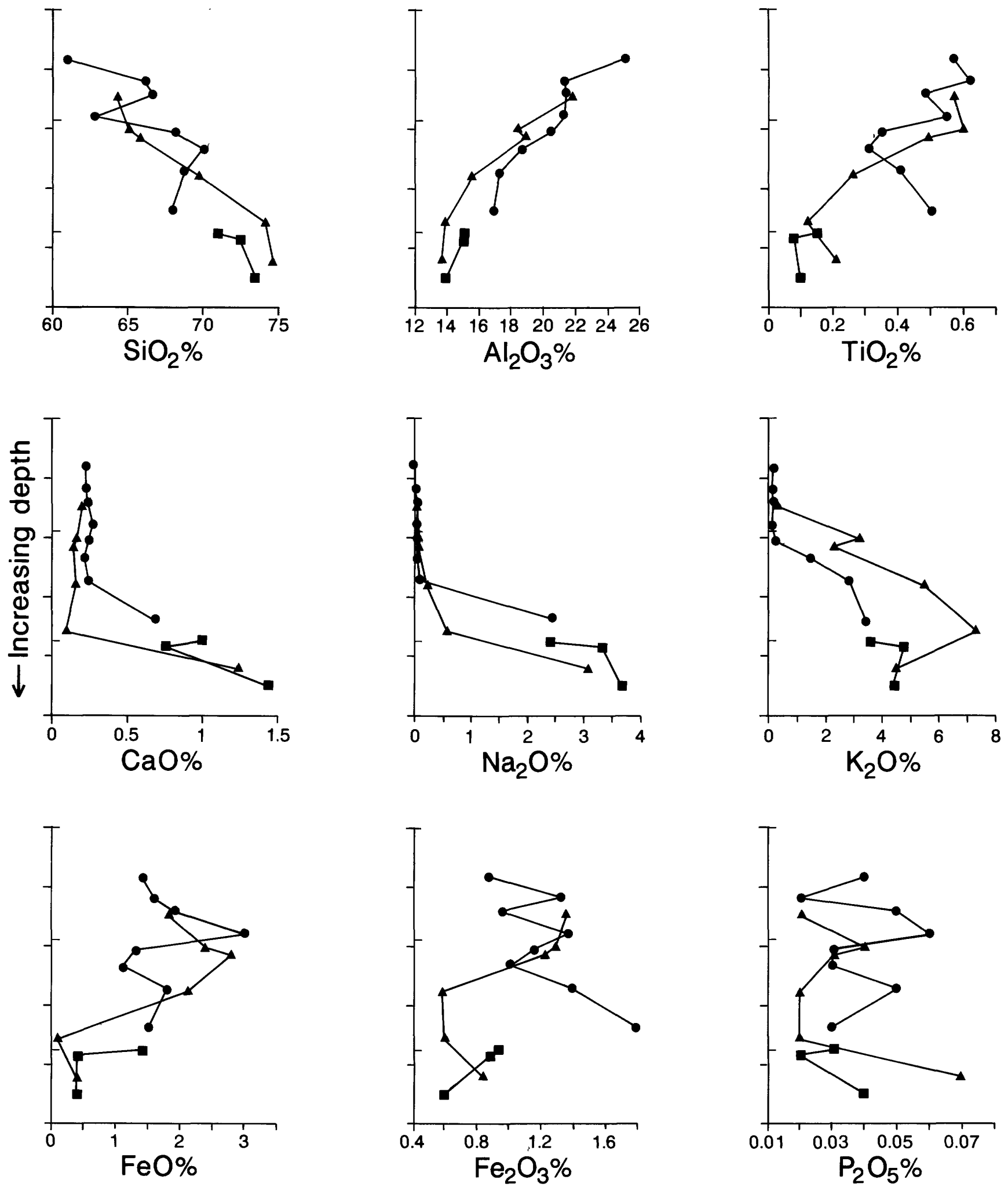

Figure H14. Whole-rock chemical trends at the Redwood Falls sites, southwestern Minnesota. The data from all three sites are arranged on a single plot and positioned according to the degree of weathering in each profile. The outside ticks on the vertical axis are 50-ft increments, and the inside ticks are 20-m increments. Square, Redwood Falls 1; triangle, Redwood Falls 2; dot, Redwood Falls 3. 

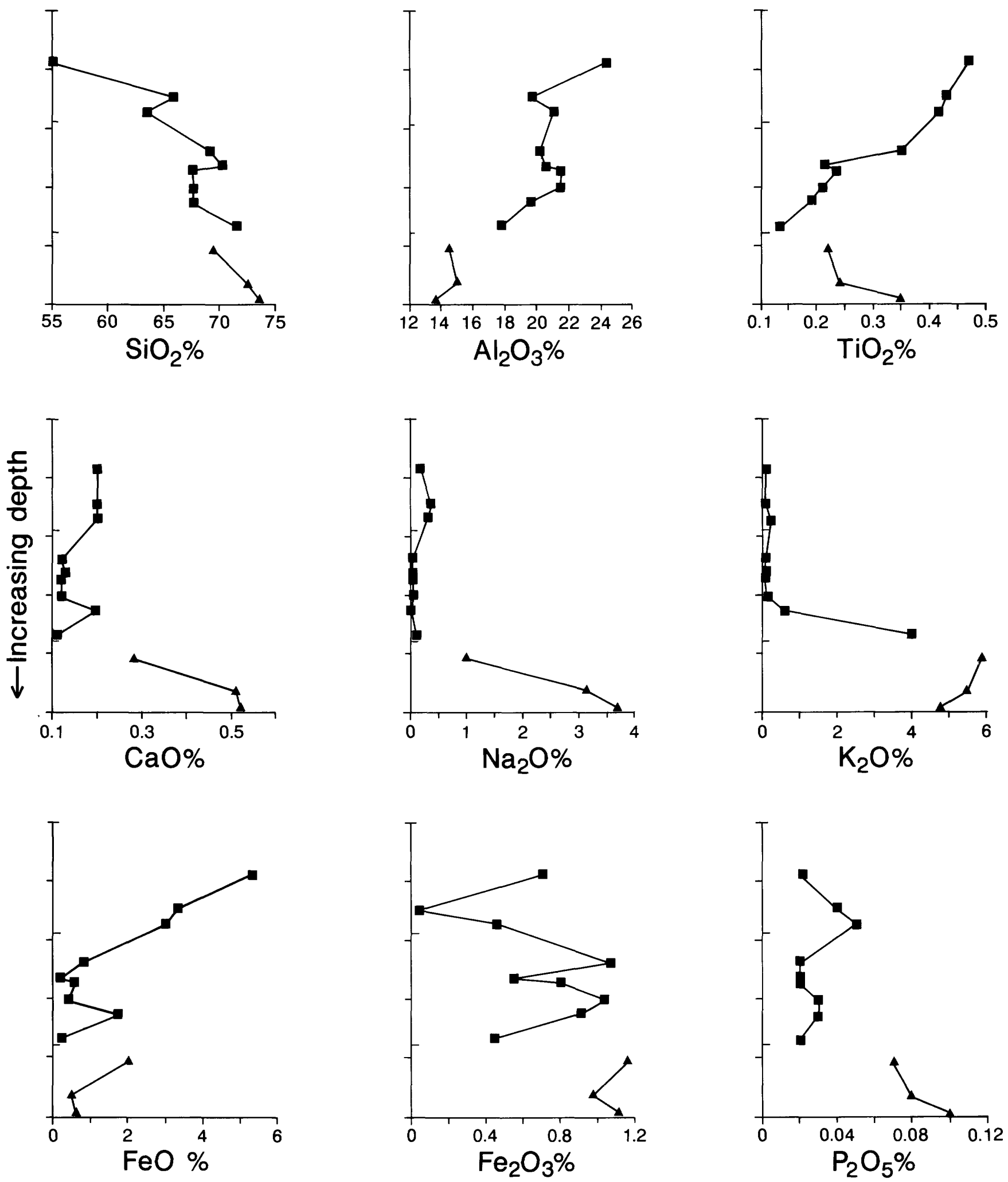

Figure H15. Whole-rock chemical trends at the Morgan sites, southwestern Minnesota. The data from both sites are arranged on a single plot and positioned according to the degree of weathering in each profile. The outside ticks on the vertical axis are 50- $\mathrm{ft}$ increments, and the inside ticks are 20-m increments. Triangle, Morgan 1; square, Morgan 2. 


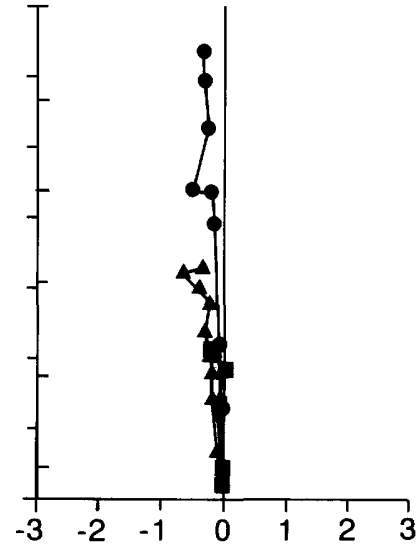

Relative concentration (log value) $\mathrm{SiO}_{2}$

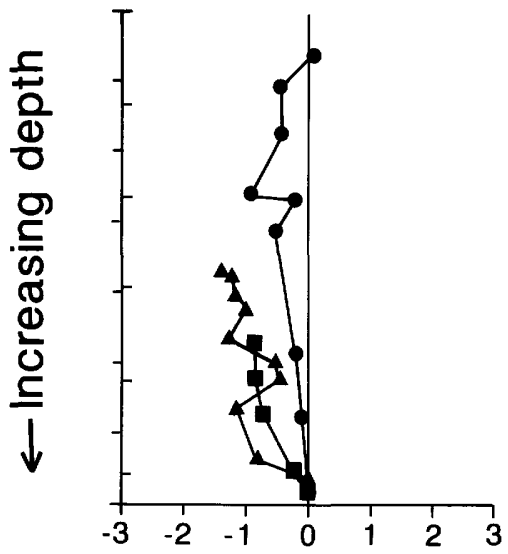

Relative concentration (log value) $\mathrm{CaO}$

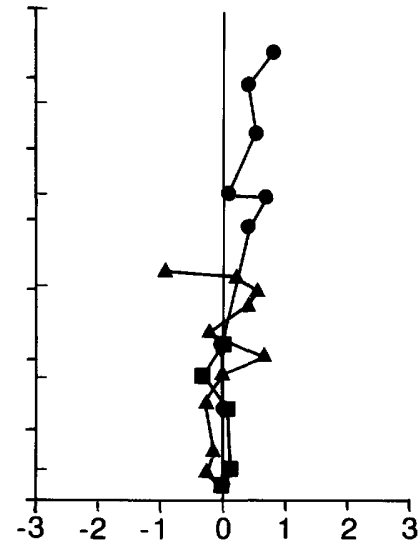

Relative concentration (log value) $\mathrm{FeO}$

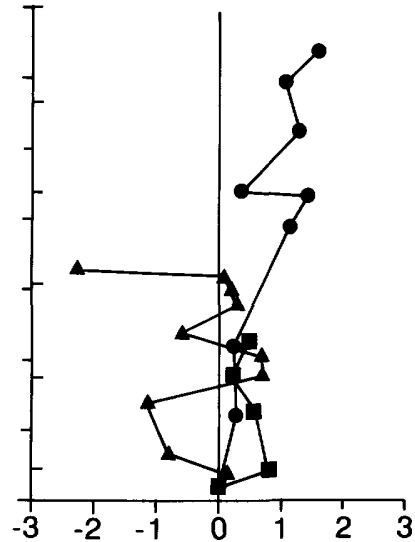

Relative concentration

(log value) $\mathrm{CO}_{2}$

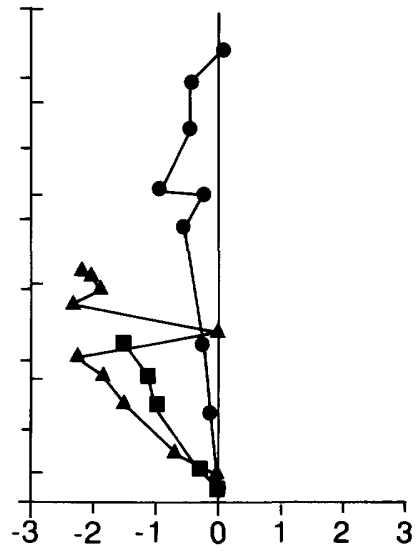

Relative concentration (log value) $\mathrm{Na}_{2} \mathrm{O}$

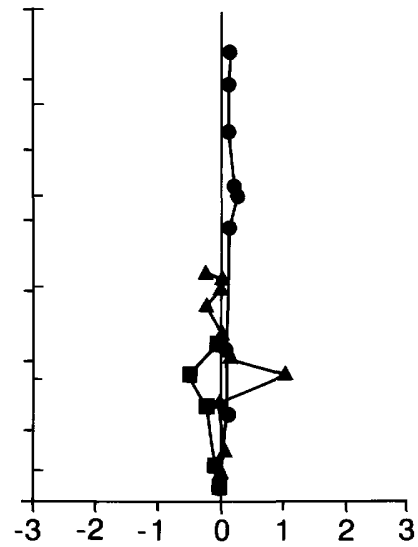

Relative concentration (log value) $\mathrm{Fe}_{2} \mathrm{O}_{3}$

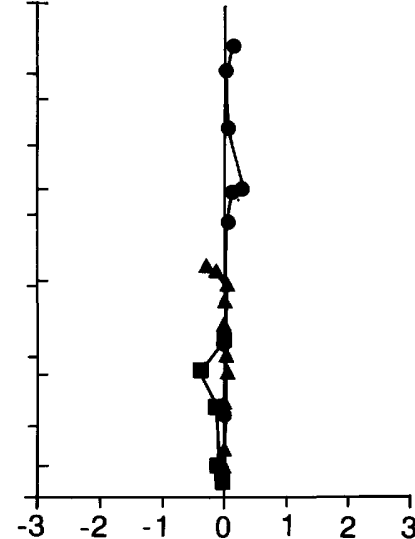

Relative concentration (log value) $\mathrm{TiO}_{2}$

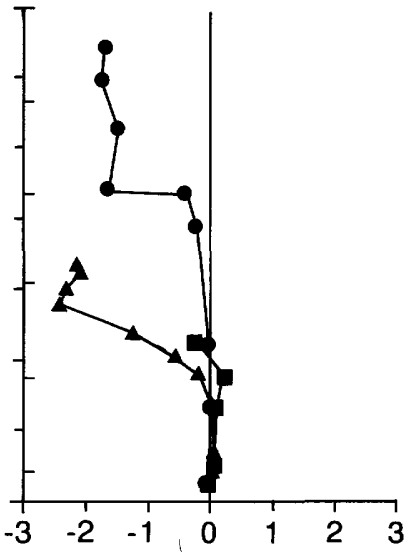

Relative concentration (log value) $\mathrm{K}$

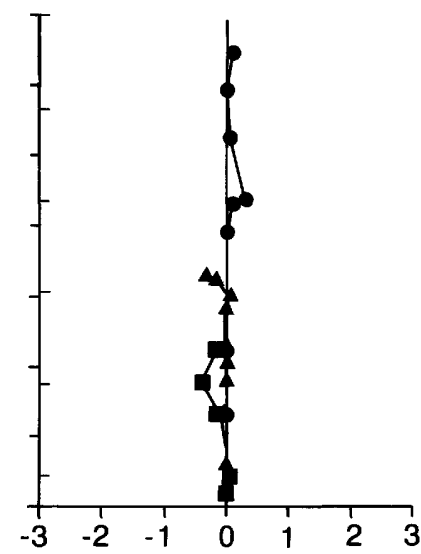

Relative concentration (log value) $\mathrm{MgO}$

Figure H16. Chemical characteristics of the St. Cloud area weathering profiles, east-central Minnesota. Based on the methods of Prasad and Roscoe (1991), where $\mathrm{Al}_{2} \mathrm{O}_{3}$ is assumed to be an inert constituent and where $\mathrm{M}_{\text {value/sample }}=$ Log $\left[\left(\mathrm{MO}_{2}\right.\right.$ sample $\left./ \mathrm{MO}_{2}{ }^{\text {parent }}\right) /\left(\mathrm{Al}_{2} \mathrm{O}_{3}\right.$ sample $\left.\left./ \mathrm{Al}_{2} \mathrm{O}_{3}{ }^{\text {parent }}\right)\right]$. The outside ticks on the vertical axis are 50 -ft increments, and the inside ticks are 20-m increments. Dot, St. Joseph; triangle, St. Augusta; square, Cold Spring. 


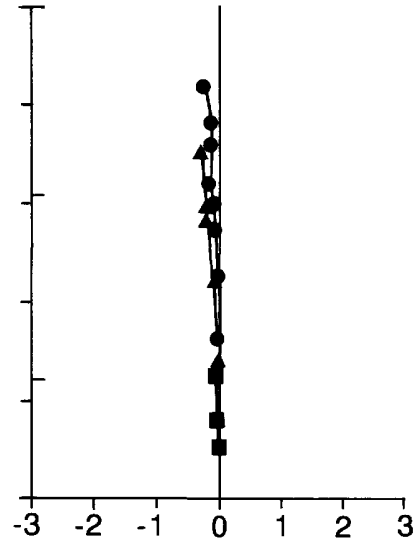

Relative concentration (log value) $\mathrm{SiO}_{2}$

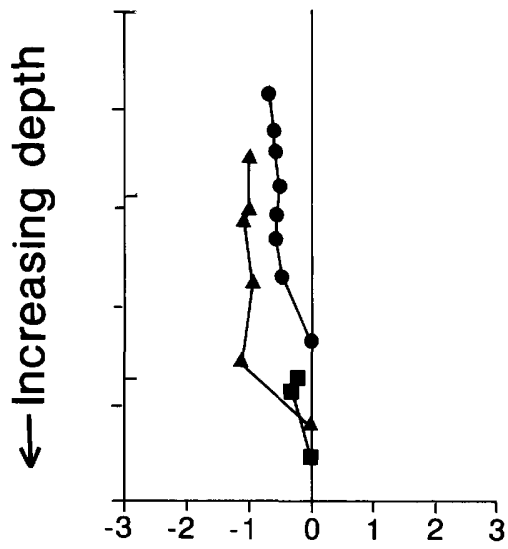

Relative concentration (log value) $\mathrm{CaO}$

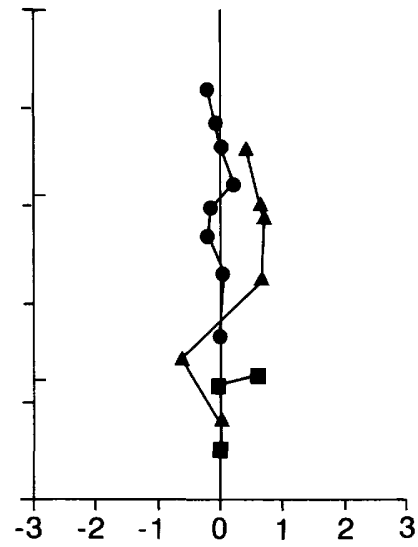

Relative concentration (log value) $\mathrm{FeO}$

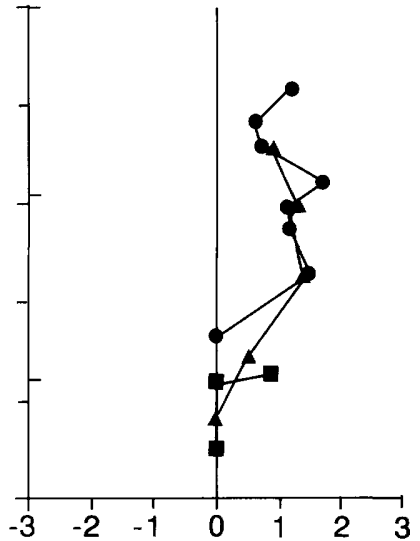

Relative concentration (log value) $\mathrm{CO}_{2}$

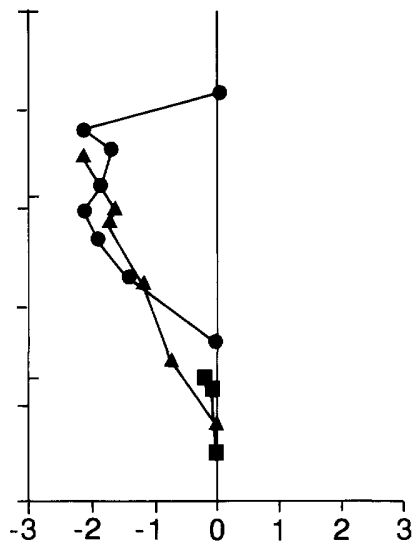

Relative concentration (log value) $\mathrm{Na}_{2} \mathrm{O}$

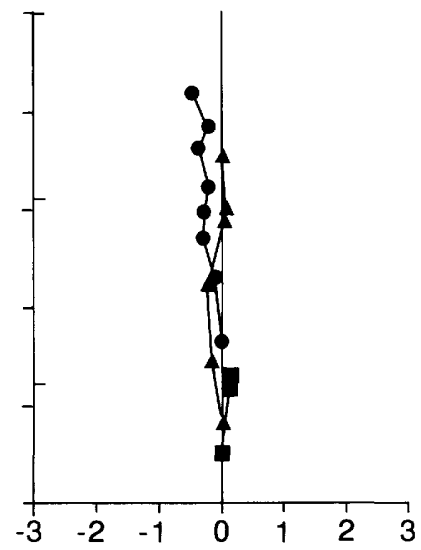

Relative concentration (log value) $\mathrm{Fe}_{2} \mathrm{O}_{3}$

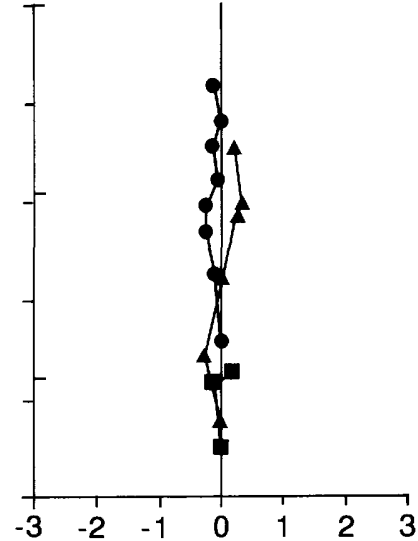

Relative concentration (log value) $\mathrm{TiO}_{2}$

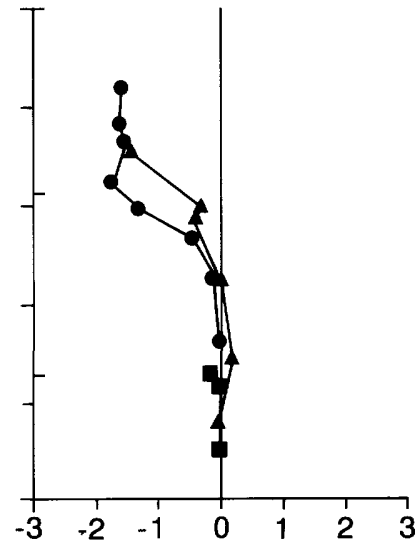

Relative concentration (log value) $\mathrm{K}_{2} \mathrm{O}$

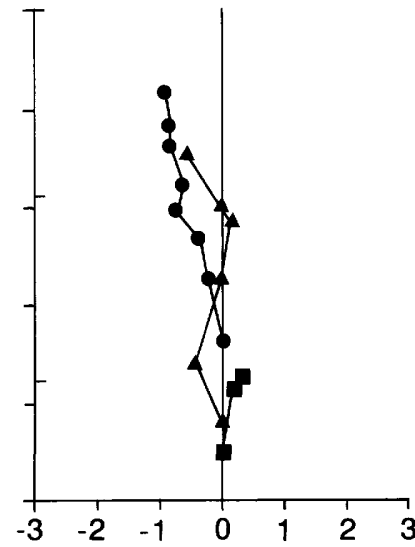

Relative concentration (log value) $\mathrm{MgO}$

Figure H17. Chemical characteristics of the Redwood Falls weathering profiles, southwestern Minnesota. Based on the methods of Prasad and Roscoe (1991), where $\mathrm{Al}_{2} \mathrm{O}_{3}$ is assumed to be an inert constituent and where $\mathrm{M}_{\mathrm{value}}$ sample $=$ Log $\left[\left(\mathrm{MO}_{2}\right.\right.$ sample $\left./ \mathrm{MO}_{2}{ }^{\text {parent }}\right) /\left(\mathrm{Al}_{2} \mathrm{O}_{3}\right.$ sample $/ \mathrm{Al}_{2} \mathrm{O}_{3}$ parent $\left.)\right]$. The outside ticks on the vertical axis are 50 -ft increments, and the inside ticks are 20-m increments. Square, Redwood Falls 1; triangle, Redwood Falls 2; dot, Redwood Falls 3. 


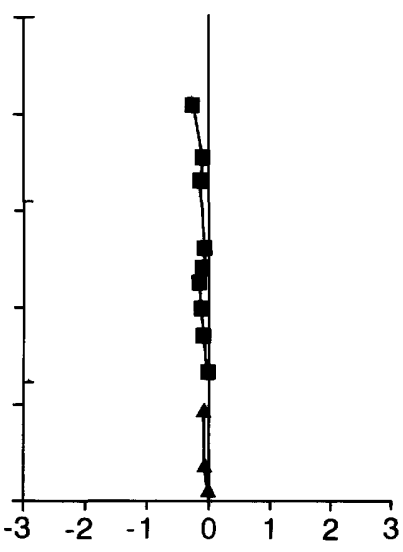

Relative concentration (log value) $\mathrm{SiO}_{2}$

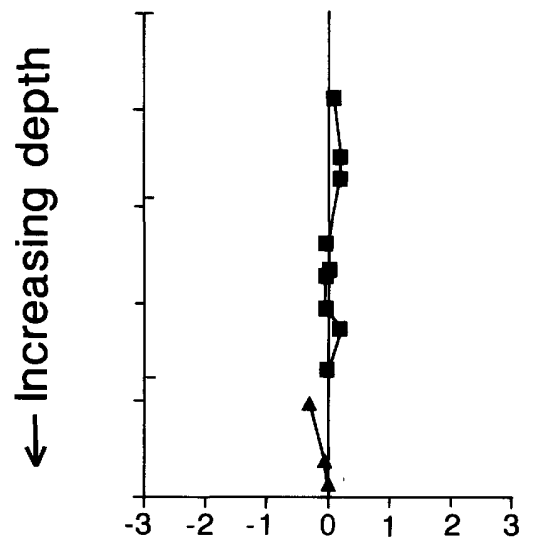

Relative concentration (log value) $\mathrm{CaO}$

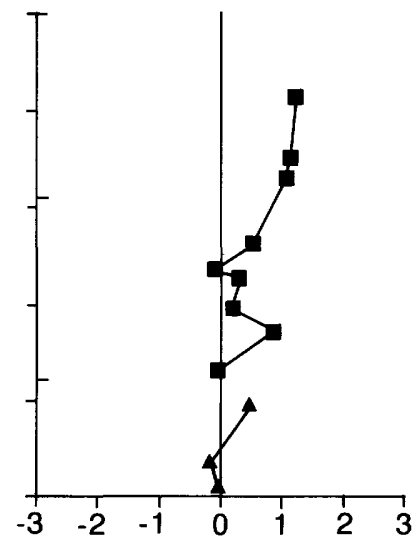

Relative concentration (log value) $\mathrm{FeO}$

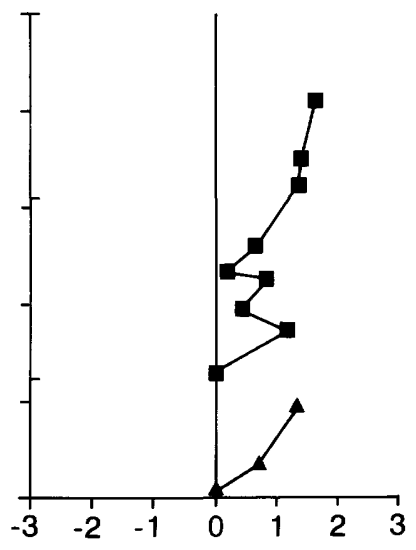

Relative concentration

(log value) $\mathrm{CO}_{2}$

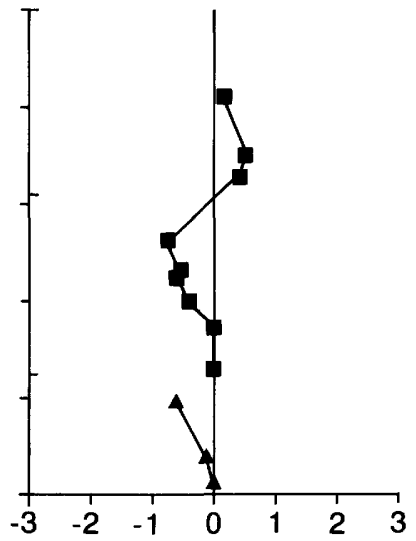

Relative concentration (log value) $\mathrm{Na}_{2} \mathrm{O}$

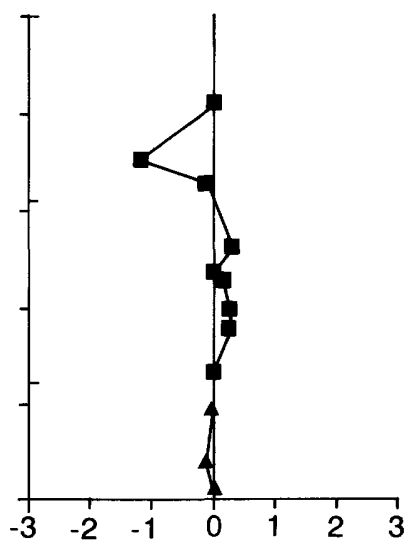

Relative concentration (log value) $\mathrm{Fe}_{2} \mathrm{O}_{3}$

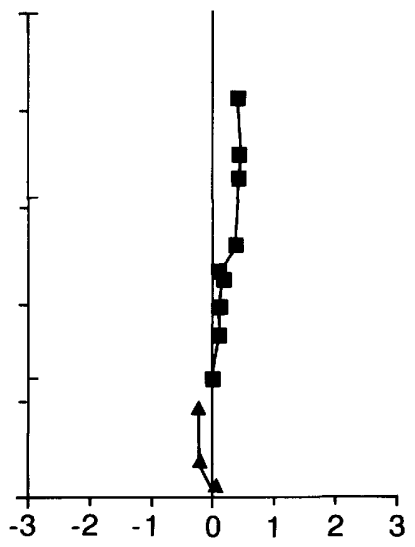

Relative concentration (log value) $\mathrm{TiO}_{2}$

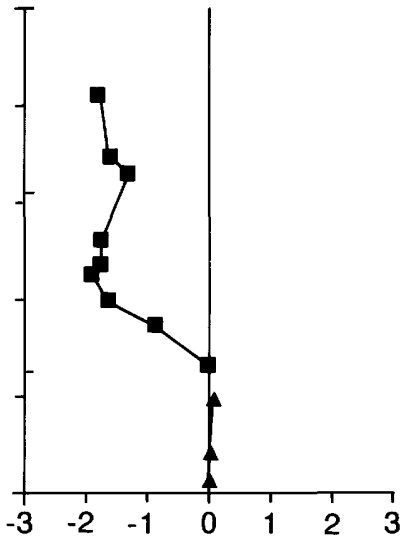

Relative concentration (log value) $\mathrm{K}_{2} \mathrm{O}$

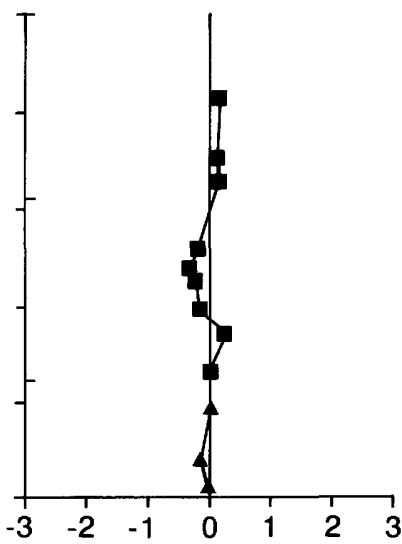

Relative concentration (log value) $\mathrm{MgO}$

Figure H18. Chemical characteristics of the Morgan 1 and Morgan 2 weathering profiles, southwestern Minnesota. Based on the methods of Prasad and Roscoe (1991), where $\mathrm{Al}_{2} \mathrm{O}_{3}$ is assumed to be an inert constituent and where $\mathrm{M}_{\mathrm{value}} /$ sample $=$ Log $\left[\left(\mathrm{MO}_{2}\right.\right.$ sample $\left./ \mathrm{MO}_{2}{ }^{\text {parent }}\right) /\left(\mathrm{Al}_{2} \mathrm{O}_{3}\right.$ sample $/ \mathrm{Al}_{2} \mathrm{O}_{3}$ parent $\left.)\right]$. The outside ticks on the vertical axis are 50 - $\mathrm{ft}$ increments, and the inside ticks are 20-m increments. Triangle, Morgan 1; square, Morgan 2. 


\section{Pisolitic Lateritic Clay}

In his regional study, Parham (1970) suggested that an interval of undisturbed pisolitic lateritic clay marks the top of the weathering profile at many places in southwestern Minnesota. However, we intersected lateritic clay only at the St. Augusta site in east-central Minnesota where it is about $1.5 \mathrm{~m}$ thick. The pisolites are hollow or are partly filled with pyrite; pyrite in turn is partly replaced by goethite. Goethite also rims many of the pisolites. A chalky, light-brownish-gray, or light-gray to white, clay-rich groundmass consists mostly of kaolinite and halloysite, and lesser amounts of quartz, gibbsite, and goethite. Siderite occurs as disseminated euhedral grains, and calcite fills many microfractures. The light-colored clay gives way downward to a green, clay-rich interval composed of kaolinite, chlorite, quartz, and lesser amounts of gibbsite and goethite. Kaolinite-rich pisolites are scattered throughout both the light-colored and green clay intervals.

\section{Chemical Attributes of the Model}

Because feldspars are a major component of the parent rocks and CIA values represent their distribution, these values are useful in correlating major chemical trends in the weathering profiles with their physical stratigraphy. Fresh rock has CIA values that vary from 47 to 53 . Although contacts are gradational, slightly weathered rock has CIA values that range from 50 to 55 , whereas saprock typically has values in the range of 55 to 65 or 70 . The lower part of the saprolith has CIA values of 67 to 82 , whereas the values in the upper part approach 95. Two samples of lateritic clay, which occurs only at the St. Augusta site, have CIA values of 97 and 98. These CIA changes represent the progressive loss of plagioclase and potassium feldspar, constituents that comprise half to twothirds of the modal volume of the parent materials. The dissolution of the feldspars and the concurrent loss of $\mathrm{CaO}, \mathrm{Na}_{2} \mathrm{O}$, and $\mathrm{K}_{2} \mathrm{O}$ from the weathering profile are well illustrated in figures H13-H15. Silica is also lost, whereas alumina, titanium, and iron content increase or remain constant.

In assessing the chemical behavior of constituents as weathering progresses, analysis must take into account changes in the bulk densities of the samples. Several schemes have been proposed to compare weight-percent chemical data from samples of different densities by normalizing the mobile constituents against some generally inert component. One procedure, developed by Reiche (1943), involves measuring progressive gains or losses of various constituents relative to the amount of alumina present in the parent material or, where that is unknown, in the least altered part of the profile (see also Prasad and Roscoe, 1991). Though unlikely to be totally immobile, alumina is one of the least mobile of the major rock-forming oxides (Goldich, 1938). Therefore, if nothing else, the procedure eliminates scatter caused by inhomogeneities in the original crystalline parents.

The chemical profiles in figures $\mathrm{H} 19-\mathrm{H} 21$ illustrate three principal upward trends: (1) the expected loss of $\mathrm{CaO}, \mathrm{Na}_{2} \mathrm{O}, \mathrm{K}_{2} \mathrm{O}$, and some $\mathrm{SiO}_{2} ;$; (2) gain of carbon dioxide; and (3) relative gains or consistency of $\mathrm{Fe}_{2} \mathrm{O}_{3}$, $\mathrm{MgO}$, and $\mathrm{TiO}_{2}$. The first trend, involving the loss of plagioclase and some loss of potassium feldspar and the concurrent loss of $\mathrm{CaO}, \mathrm{Na}_{2} \mathrm{O}, \mathrm{K}_{2} \mathrm{O}$, and possibly some $\mathrm{SiO}_{2}$, has been discussed. However the data in figures $\mathrm{H} 16$, $\mathrm{H} 17$, and H18 show that sodium is lost from the system much earlier than calcium. Thus, the loss of sodium must be the rate-controlling step in the dissolution of plagioclase. The data also show that at least some calcium remains in the system, where it becomes available to combine with carbon dioxide to form calcite, typically in the upper part of the weathering profiles. Potassium is lost from the system at a stratigraphic level somewhat above the level at which calcium and sodium are lost. This is consistent with the relatively greater stability of potassium feldspar, and with the development of illite as an intermediate weathering product. Potassium, like sodium, is generally lost at the top of the saprolith. Some potassium is apparently gained at the very top of the profile at the St. Joseph site, in the St. Cloud area.

Iron and magnesium behave somewhat differently in the profiles. In general, ferrous iron is more or less constant in the lower half, but increases slightly in the upper half of several profiles. This trend corresponds to the dissolution of hornblende and biotite and the concurrent release of ferrous iron, which migrates upward to a level where oxidizing conditions fix it as either goethite or hematite. Several profiles also show a slight decrease in ferric iron in their uppermost parts. Solutions moving downward in the vadose zone may dissolve small amounts of ferric iron and reprecipitate it in the phreatic zone. Magnesium shows a much more erratic distribution. In several profiles, it systematically declines from bottom to top, whereas in others it remains more or less constant through the lower half and declines abruptly in the upper half of the saprolithic interval. That abrupt decline generally corresponds to the level where biotite disappears.

Techniques developed by Nesbitt and Young (1984, 1989) further demonstrate the chemical changes that occur during weathering. They have shown that chemical trends in modern weathering profiles developed on granitic rocks mimic those predicted from kinetic leaching data for alkali 

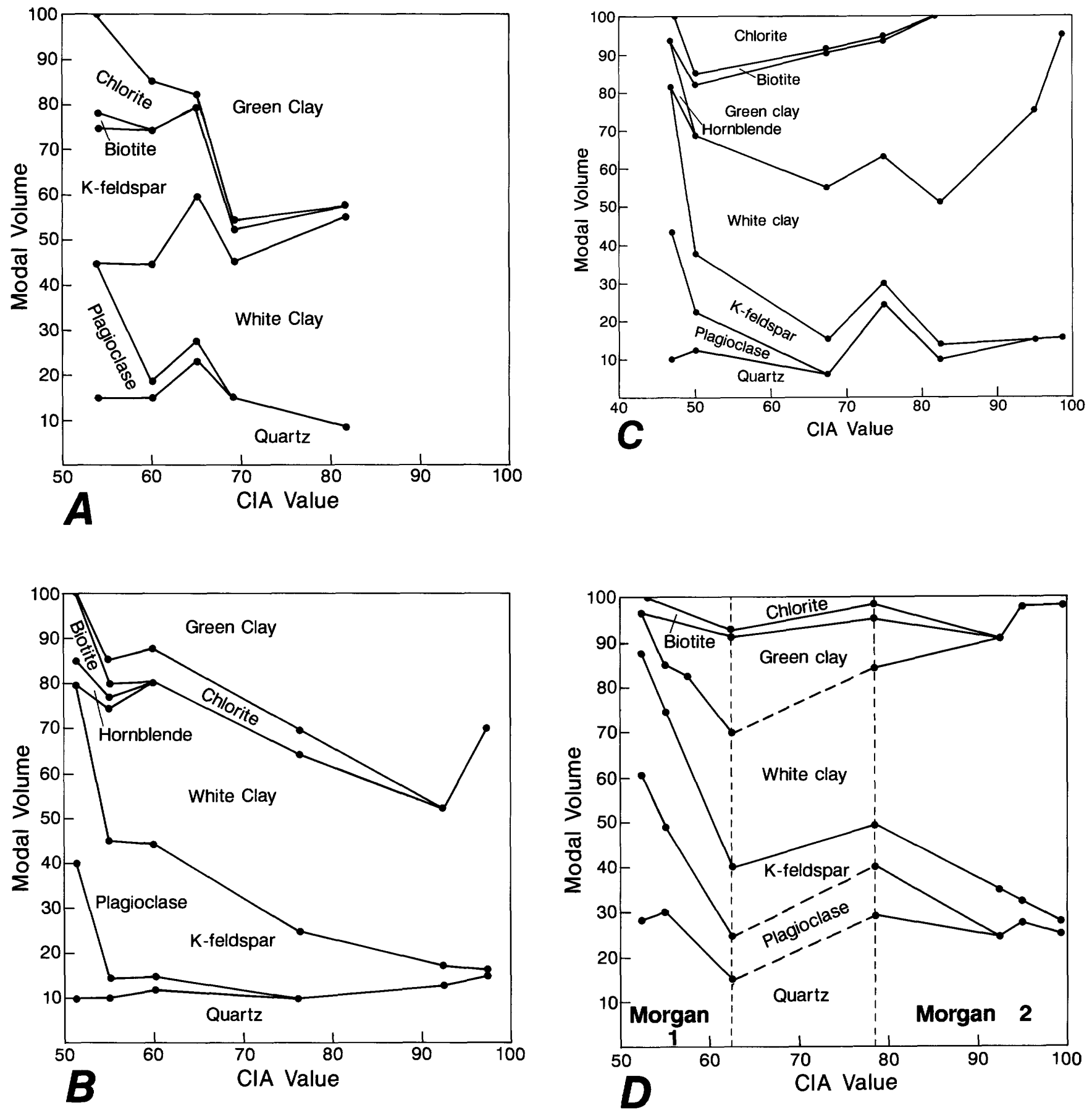

Figure H19 (above and facing page). Plots of modal proportions of mineral constituents in samples, ordered by $\mathrm{ClA}$ (chemical index of alteration; Nesbitt and Young, 1982) values. Each vertical series of dots represents a sample aligned on its CIA value. The width of each field along that vertical line corresponds to the proportion of the constituent named in the field. Modal proportions determined from thin sections and visual estimation from core. $A$, Cold Spring; $B$, St. Joseph; $C$, St. Augusta; $D$, Morgan 1 and Morgan 2; E, Redwood Falls 2 and Redwood Falls 3; F, Redwood Falls 1 . For each sample, total proportion of mineral constituents equals 100 . 

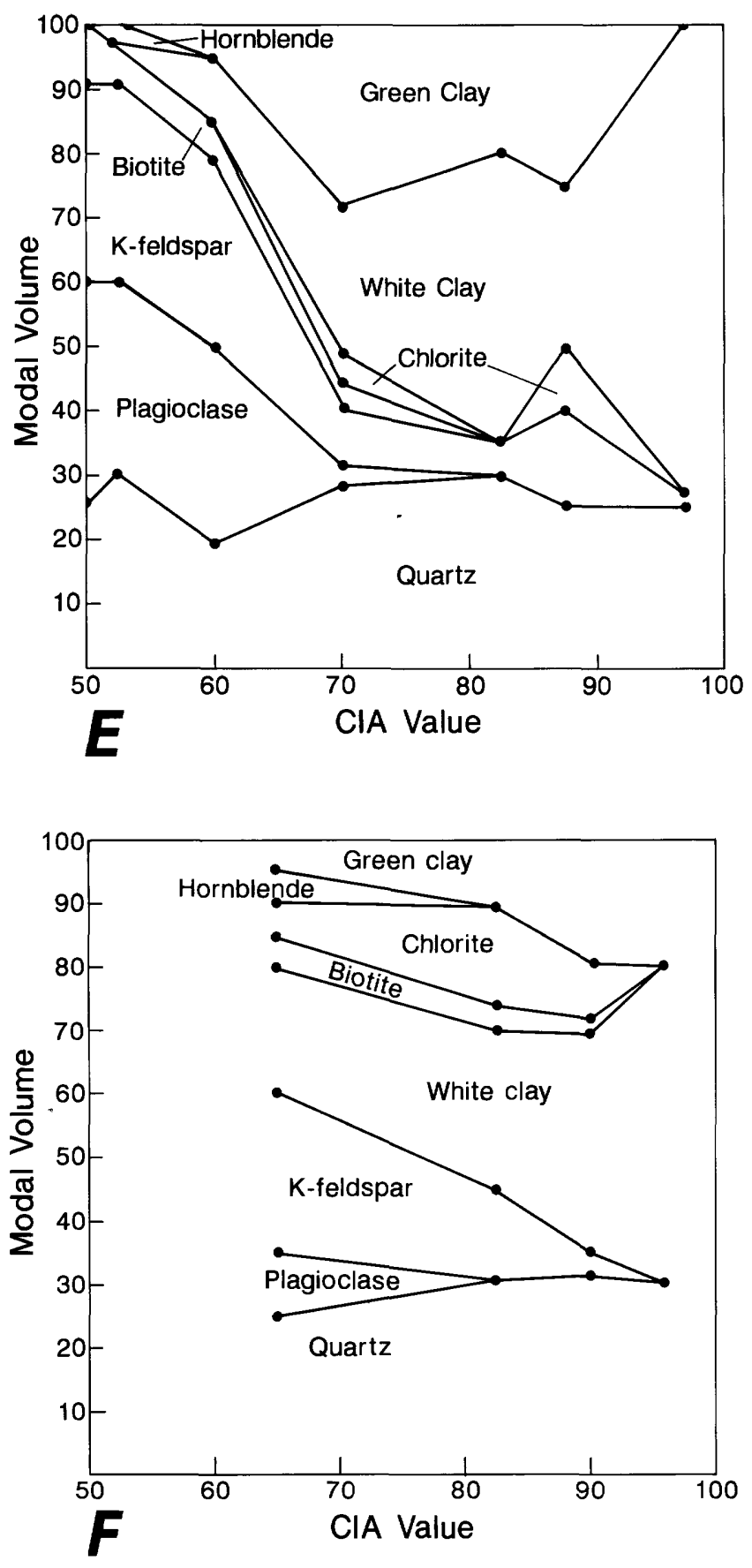

and calcium from feldspars. Ternary diagrams in the field $\mathrm{Al}_{2} \mathrm{O}_{3}-\mathrm{CaO}+\mathrm{Na}_{2} \mathrm{O}-\mathrm{K}_{2} \mathrm{O} \quad(\mathrm{A}-\mathrm{CN}-\mathrm{K})$ show that the progressive dissolution of plagioclase moves the bulk composition of the residue toward the $\mathrm{A}-\mathrm{K}$ join (fig. H21). Residues located near that join are poor in calcium- or sodium-bearing phases, and the predominant remaining minerals include aluminous phases, such as kaolinite, and potassic phases, such as potassium feldspar and illite. As weathering proceeds, the trend is abruptly redirected toward the alumina apex, reflecting the dissolution of the potassic phases and the concurrent increase in the profile of residues enriched in the aluminum-bearing phases. Similarly, on an $\mathrm{Al}_{2} \mathrm{O}_{3}-\mathrm{CaO}+\mathrm{NaO}^{-}+\mathrm{K}_{2} \mathrm{O}-\mathrm{FeO}+\mathrm{MgO}$ (A-CNK-FM) diagram, the initial trend during early weathering moves the residual compositions in a direction sub-parallel to the $\mathrm{A}-\mathrm{CNK}$ join. As the $\mathrm{A}-\mathrm{FM}$ join is approached during advanced weathering, there is again an abrupt change in the compositional trend toward the alumina apex. This relationship implies that iron and magnesium are leached from the residual products more rapidly than is alumina. Nesbitt and Young (1984) suggest that these trends can be used as templates to which the chemical history of geologically old profiles can be compared. Deviations from the templates generally are caused by heterogeneity in the protolith composition, or post-weathering diagenetic modifications. For example, four trends are apparent in the St. Joseph data (fig. H21B). The first, involving samples $9,8,7$, and 6 , defines compositional changes associated with the weathering of granite. The second, apparent on the $\mathrm{A}-\mathrm{CN}-\mathrm{K}$ diagram, shows that the upper part of the saprolith is enriched in the CN component. The third shows that the uppermost part of the saprolith is also enriched in the FM component. The second and third trends are most likely the result of diagenetic processes that added or concentrated constituents such as calcium and iron to the upper part of the profile. The fourth trend, involving samples 4 and 5 , is associated with the weathering of a diabasic protolith.

Similar bulk compositional changes are seen at the St. Augusta site. In the $\mathrm{A}-\mathrm{CN}-\mathrm{K}$ diagram of figure $\mathrm{H} 21 C$, materials derived from a granitic protolith (samples 11-8 and 5) define a weathering trend in which the saprolith is enriched in alumina at the expense of alkalies and calcium. Samples 3, 2, and 1 are lateritic clays that cluster at the alumina apex. A trend involving enrichment in alumina at the expense of iron and magnesium is also well illustrated on the $\mathrm{A}-\mathrm{CNK}-\mathrm{FM}$ diagram. which shows that samples 3-1 follow a trend parallel to the A-FM join. Materials of diabasic affinity (samples 7, 6, and 4) define a second weathering trend.

In another example (fig. $\mathrm{H} 21 E$ ), the partial profiles at Morgan 1 and Morgan 2 together define a similar trend showing the progressive loss of calcium at Morgan 1 and the progressive loss of potassium at Morgan 2. However, the upper part of the Morgan 2 profile is unique in that it is apparently enriched in sodium or calcium such that compositional changes follow the $\mathrm{A}-\mathrm{CN}$ join toward the $\mathrm{CN}$ apex. That part of the profile also shows appreciable iron enrichment on an $\mathrm{A}-\mathrm{CNK}-\mathrm{FM}$ plot. It is therefore likely that the upper part of the profile at Morgan 2 was affected by post-weathering diagenetic processes.

At Redwood Falls 2 (fig. H21F) samples 6, 4, 3, 2, and 1 define an ordinary weathering sequence in both the $\mathrm{A}-\mathrm{CN}-\mathrm{K}$ and $\mathrm{A}-\mathrm{CNK}-\mathrm{FM}$ diagrams. Sample 5, a pegmatitic segregation, falls off that trend in both diagrams. 


\section{SUMMARY}

In parts of western Minnesota a weathering profile, as much as $90 \mathrm{~m}$ thick, was developed on a variety of prePhanerozoic igneous and metamorphic rocks (Parham, 1970). In the areas described here, evidence of weathering extends in places to a depth of at least $60 \mathrm{~m}$ beneath the paleogeomorphic land surface. The generalized weathering profile and landform model illustrated in figure $\mathrm{H} 22$ shows that weathering occurred along fractures and joints as shown by isolated corestones separated by considerably altered material. In general, some quartz and at least trace amounts of potassium feldspar persist throughout most of the profiles. Plagioclase is rapidly weathered with the
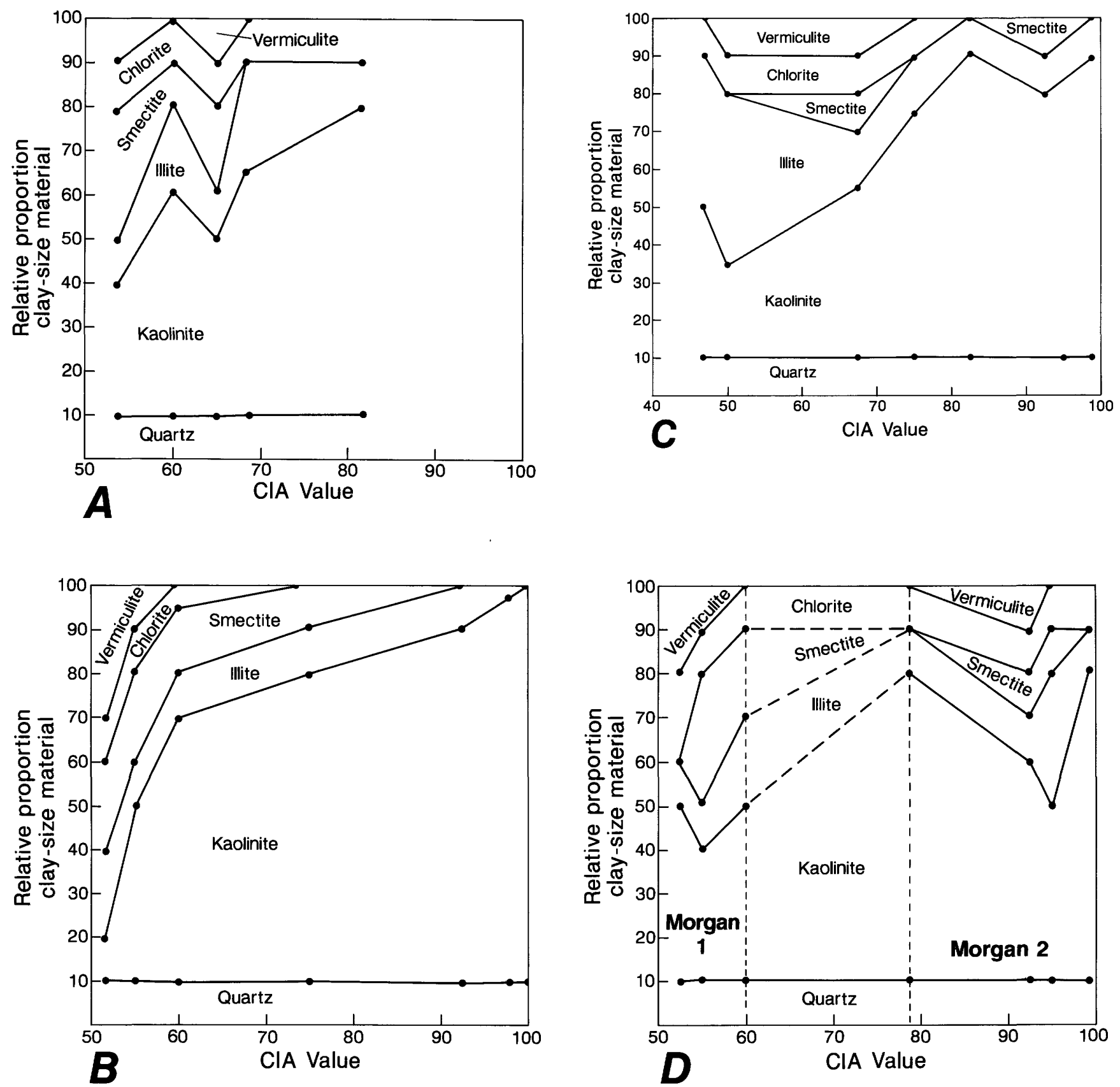

Figure $\mathbf{H} 20$ (above and facing page). Plots of relative proportions of clay-sized material versus CIA (chemical index of alteration; Nesbitt and Young, 1982). Each vertical series of dots represents a sample aligned on its CIA value. The width of each field along that vertical line corresponds to the proportion of the constituent named in the field. Relative proportions determined by $\mathrm{X}$-ray diffraction of clay-sized material. A, Cold Spring; B, St. Joseph; $C$, St. Augusta; D, Morgan 1 and Morgan 2; E, Redwood Falls 2 and Redwood Falls 3; F, Redwood Falls 1. 

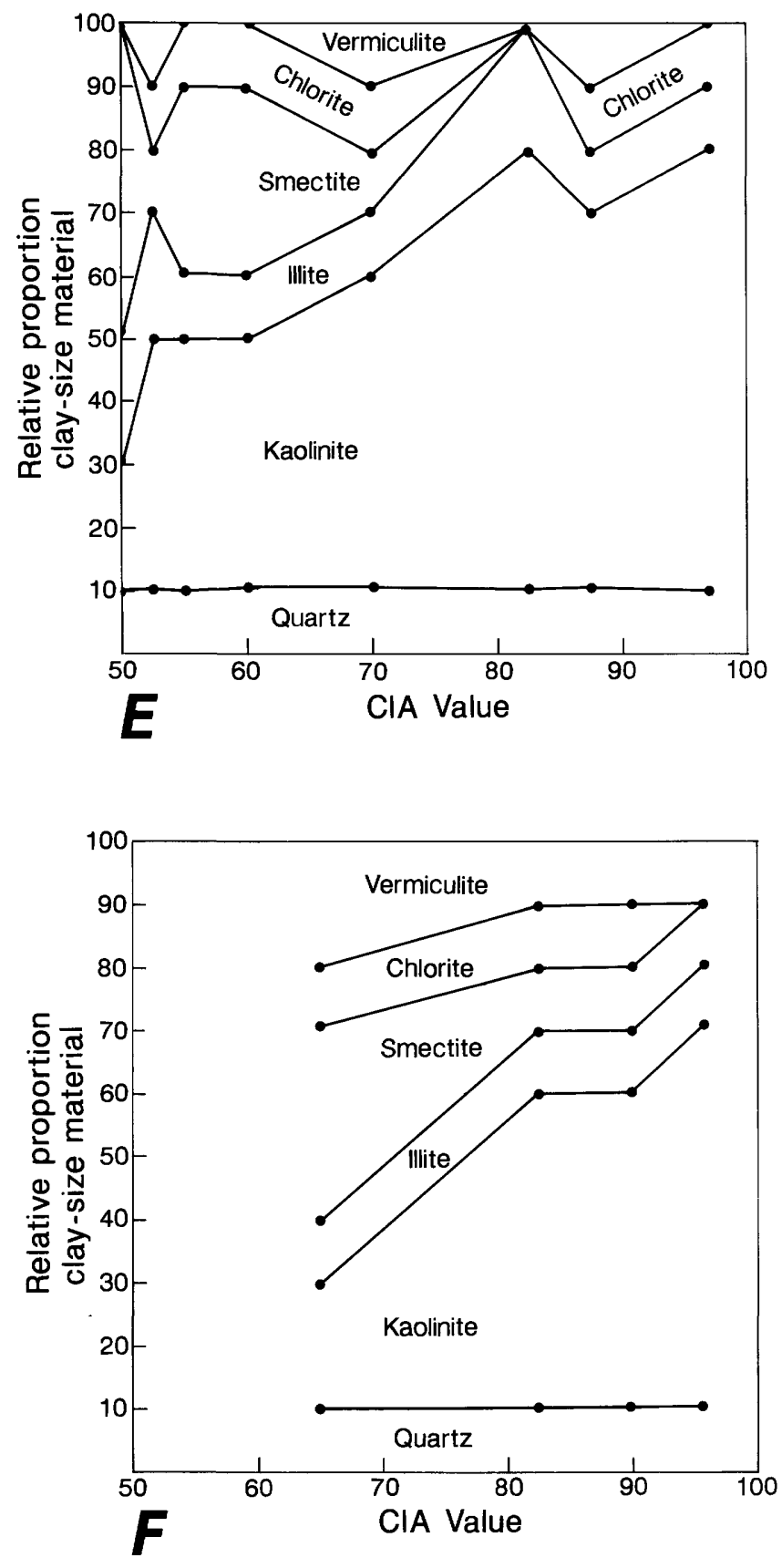

concurrent and pronounced loss of calcium and sodium. Large amounts of kaolinite are ultimately produced at the expense of the plagioclase. As weathering proceeds, illite forms as a common alteration product of potassium feldspar, which dissolves much more slowly than plagioclase. However, with continued weathering and the concurrent loss of potassium, the illite is transformed into kaolinite. Hornblende and biotite alter to chlorite, vermiculite, iron oxides, and other clay minerals such as the smectites, and at the same time aggressive solutions reacting with these phases produce alumina-rich phases such as kaolinite and, less commonly, gibbsite.
The extremely weathered upper parts of well-developed profiles, which contain abundant kaolinite, have been the focus of the exploration community. Other factors, however, tend to complicate that simple relationship. In many places the weathering profiles have been modified by diagenetic processes involving the remobilization of iron, calcium, and carbon dioxide to produce very late forming phases such as siderite, calcite, hematite or goethite, and pyrite. Unlike the in-situ weathering products formed by through-flowing ground-water solution, these minerals likely accumulated at some later time in poorly drained areas under stagnant conditions, and their presence may be a useful indicator of the changing position of the water table in pre-Late Cretaceous time. From petrographic and X-ray diffraction data it is not possible to distinguish mineral phases that formed by in-situ weathering or diagenetic processes from those that formed much later by metasomatic processes involving the introduction of chemical constituents from outside the weathering system. For example, the lateritic clay interval at St. Augusta and the upper part of the saprolithic intervals at Redwood Falls 1 and Morgan 2 are apparently enriched in sodium, a component which is relatively mobile under ordinary weathering conditions. However such situations are not unique, and Nesbitt and Young (1989) suggest that sodium metasomatism can occur, mainly by reactions involving the weathering profile and marine water having a large ratio of sodium to potassium. If so, the weathering profile in western Minnesota records the incursion of a marine sedimentary system during Late Cretaceous time.

\section{ECONOMIC IMPLICATIONS}

Potential users of the in-situ kaolinite resource in Minnesota require a thick, widespread kaolinite-rich product, free of impurities that would tend to degrade the quality. Ideally, the uppermost part of the weathering profile, where intense chemical weathering has reduced the saprolith to a mixture of kaolinite and quartz, would best satisfy a user's needs. However, a breakdown in the mechanical integrity of that part of the weathering profile has led to an environment particularly susceptible to the introduction of diagenetic and metasomatic constituents that will contaminate the deposits. Thus, it seems likely that if the saprolith is to be fully utilized as a kaolinite resource, at least some beneficiation will be necessary. This problem, which has plagued the exploration community for almost as long as the resource has been recognized, has been examined in considerable detail by Wayland (1964), and more recently by Prasad and Reid (1989).

Although problems persist, contemporary mineral exploration in Minnesota is tackling increasingly more difficult areas lacking significant outcrop. This is in part 

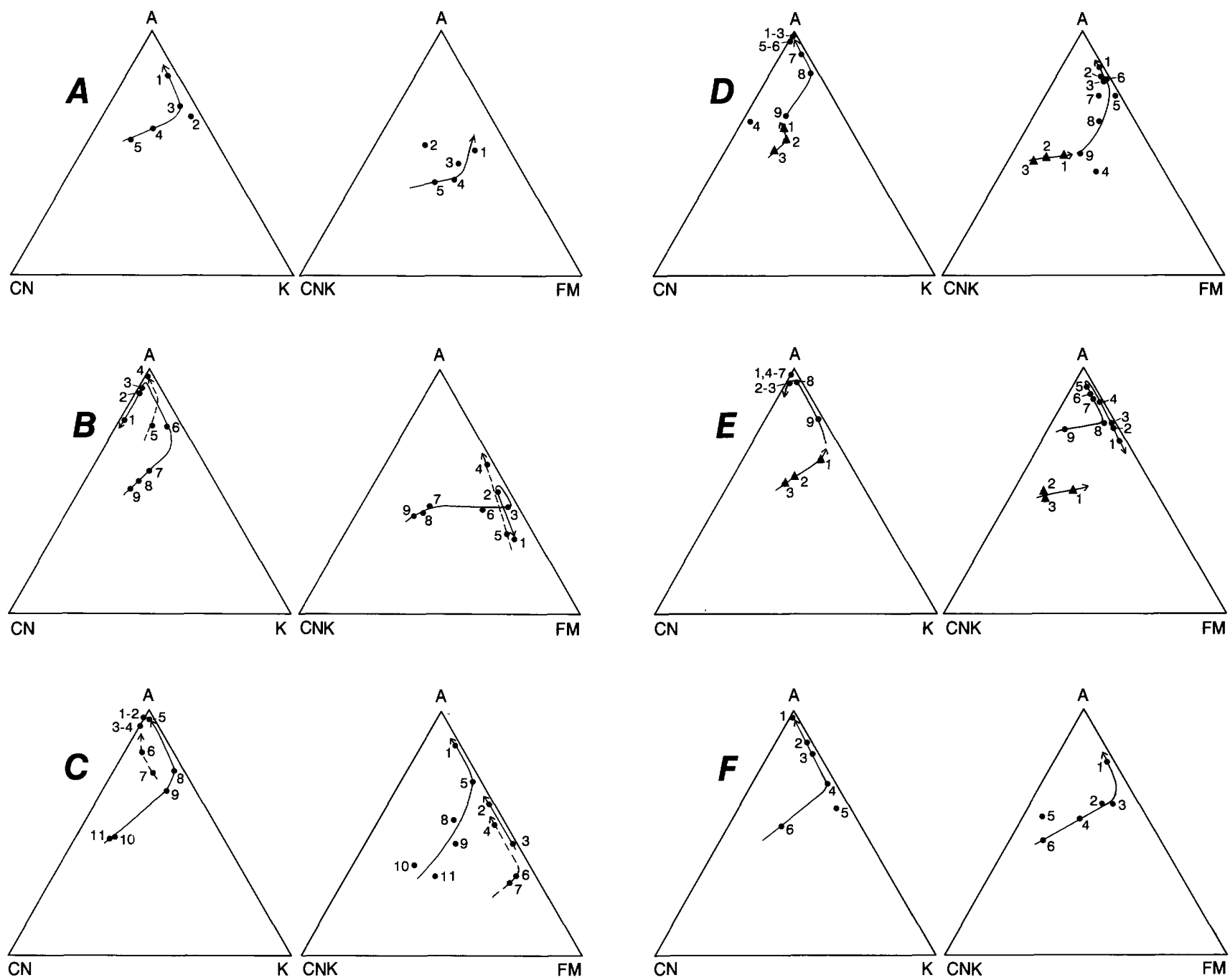

Figure H21. Ternary diagrams in the $\mathrm{Al}_{2} \mathrm{O}_{3}-\mathrm{CaO}+\mathrm{Na}_{2} \mathrm{O}-\mathrm{K}_{2} \mathrm{O}$ and $\mathrm{Al}_{2} \mathrm{O}_{3}-\mathrm{CaO}+\mathrm{Na}_{2} \mathrm{O}+\mathrm{K}_{2} \mathrm{O}-\mathrm{FeO}+\mathrm{MgO}$ fields showing chemical trends in the weathering of granitic rocks. $A$, Cold Spring; $B$, St. Joseph; $C$, St. Augusta; $D$, Redwood Falls 1 and Redwood Falls 3 (triangle); E, Morgan 1 (triangle) and Morgan 2; F, Redwood Falls 2. The numbers are those used in figures $\mathrm{H} 5-\mathrm{H} 12$ to indicate sample locations in the drill core. The samples were numbered consecutively from the top of the core to the bottom.

for the obvious reason that many areas having reasonable outcrop have already received exploration attention, even by several generations of activity. Another reason is that it has been shown that the non-outcropping areas contain depositional regimes characterized by widespread kaolinite-rich sediments reworked from saprolith and redeposited as parts of a fluvial system that existed prior to the incursion of marine water in Late Cretaceous time (Hauck and Heine, 1991). Some sedimentary kaolinitic deposits are at least $14 \mathrm{~m}$ thick in some channels or valleys. Although they thin rapidly away from the valleys, they probably remain relatively uniform in thickness for some distance along the strike of the channels. Clearly, these reworked deposits are inviting exploration targets, but it will be necessary to establish a better understanding of weathering profile and landform relationships in western Minnesota to find and delineate them.

Lastly, this study has emphasized weathering processes that affect rocks of quartzofeldspathic composition. However, much of the area where saprolith is developed in western Minnesota is also underlain by mafic volcanic rocks of Archean age. As judged from numerous mines just across the International Boundary, in Ontario, these so-called "greenstone belts" have potential for lode-gold and base-metal (zinc and copper) deposits. Exploration in the greenstone belts has focused on finding a primary deposit, but it is interesting to speculate about the size and grade of a world-class Archean deposit that was modified by supergene processes related to an episode of intense weathering before the arrival of Late Cretaceous seas. 

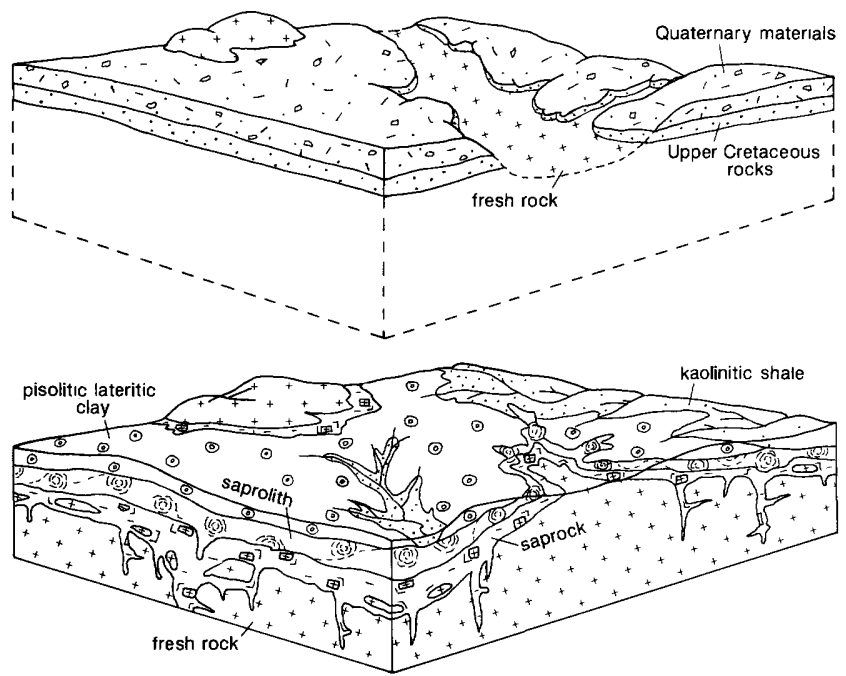

Figure H22. Physical stratigraphy of the in-situ and reworked weathering products. The patterns (as in figs. $\mathrm{H} 5-\mathrm{H}_{1}$ ) in the lower block diagram represent fresh rock, saprock, saprolith, pisolitic lateritic clay, and fluvial deposits of kaolinitic shale. Upper Cretaceous and (or) Quaternary deposits (upper block) cover this profile in most places.

\section{REFERENCES CITED}

Austin, G.S., 1970, Weathering of the Sioux Quartzite near New Ulm, Minnesota, as related to Cretaceous climates: Journal of Sedimentary Petrology, v. 40, no. 1, p. 184-193.

Bickford, K.F., and Price, D., 1947, Report on work done on clays in summer 1947: Unpublished Minnesota Geological Survey file report, $20 \mathrm{p}$.

Chesworth, W., 1992, Weathering systems, Ch. 2 in Martini, I.P., and Chesworth, W., eds., Weathering, soils, and paleosols: New York, Elsevier, p. 19-40.

Dott, R.H., Jr., and Batten, R.L., 1981, Evolution of the Earth (3d ed.): New York, McGraw-Hill, 573 p.

Goldich, S.S., I938, A study in rock weathering: Journal of Geology, v. 46, p. $17-58$.

Grout, F.F., and Soper, E.K., I919, Clays and shales of Minnesota: U.S. Geological Survey Bulletin 678, 259 p.

Hall, C.W., 1899, The gneisses, gabbro schists, and associated rocks of southwestern Minnesota: U.S. Geological Survey Bulletin 157, 160 p., includes maps.

Hall, C.W., Meinzer, O.E., and Fuller, M.L., 19I1, Geology and underground waters of southern Minnesota: U.S. Geological Survey Water Supply Paper 256, p. 34-35.

Hauck, S.A., and Heine, J.J., 1991, Regional and local geologic, mineralogic, and geochemical controls of industrial clay grades in the Minnesota River Valley and the Meridian aggregates quarry, St. Cloud, Minnesota: University of Minnesota, Natural Resources Research Center Technical Report NRRI/TR-91/15.

Hauck, S., Heine, J.J., Zanko, L., Power, B., Geerts, S., Oreskovich, J., and Reichoff, J., 1990, LCMR clay project; NRRI summary report: University of Minnesota, Natural Resources Research Institute Technical Report NRRI/GMIN-TR-89-12A, 201 p., 7 pls.
Moore, W.L., 1979. A preliminary report on the Red River Valley drilling project, eastern North Dakota and north-western Minnesota: U.S. Department of Energy Open-File Report GJBX-3(79), 292 p.

Morey, G.B., 1983, Animikie basin, Lake Superior region, U.S.A., in Trendall, A.F., and Morris, R.C., eds., Iron-formation facts and problems: Amsterdam, Elsevier, p. 13-67.

Mossler, J.H., 1978, Results of subsurface investigations in northwestern Minnesota, 1972: Minnesota Geological Survey Report of lnvestigations 19, $18 \mathrm{p}$.

Nesbitt, H.W., and Young. G.M., 1982, Early Proterozoic climates and plate motions inferred from major element chemistry of lutites: Nature, v. 299, p. 715-717.

1984, Prediction of some weathering trends of plutonic and volcanic rocks based on thermodynamic and kinetic considerations: Geochimica et Cosmochimica Acta, v. 48, p. 1523-1534.

1989, Formation and diagenesis of weathering profiles: Journal of Geology, v. 97, p. 129-147.

Parham, W.E., 1970, Clay mineralogy and geology of Minnesota's kaolin clays: Minnesota Geological Survey Special Publication Series SP-10, 142 p.

Prasad, M.S., and Reid, K.J., 1989, Process options and testing procedures for Minnesota clays: Mineral Resources Research Center Technical Report MRRC/LCMR-TR-89-08.

Prasad, M.S., and Roscoe, S.M., 1991, Profiles of altered zones at ca $2.45 \mathrm{Ga}$ unconformities beneath Huronian strata, Elliot Lake. Ontario: Evidence for early Aphebian weathering under anoxic conditions, in Current research, pt C: Geological Survey of Canada Paper 91-1C, p. 43-54.

Reiche, P., 1943, Graphic representation of chemical weathering: Journal of Sedimentary Petrology, v. 13, p. 58-68.

Setterholm, D. R., 1994, The Cretaceous rocks of southwestern Minnesota - A marine to nonmarine transition along the eastern margin of the Western Interior Seaway, in Shurr, G.W., and Hammond, R.H., eds., Geology of the eastern margin of the Western Interior Seaway: Geological Society of America Special Paper 287, p. 97-110.

Setterholm, D.R., Morey, G.B., Boerboom, T.J., and Lamons, R.C., 1989, Minnesota kaolin clay deposits-A subsurface study in selected areas of southwestern and east-central Minnesota: Minnesota Geological Survey Information Circular 27, $99 \mathrm{p}$.

Streckeisen, A., 1976, To each plutonic rock its proper name: Earth Science Reviews, v. 12, p. 1-35.

Symons, D.T.A., 1966, A paleomagnetic study on the Gunflint, Mesabi, and Cuyuna iron ranges in the Lake Superior region: Economic Geology, v. 61, no. 8, p. 1336-1361.

Tyler, S.A., and Bailey, S.W., 1961, Secondary glauconite in the Biwabic iron-formation of Minnesota: Economic Geology, v. 56, no. 6 , p. $1033-1044$.

Upham, W., 1888, The geology of Renville County: Minnesota Geological Survey Final Report, v. 2, p. 190-204.

Wayland, T.E., 1964, The Redwood Falls kaolin deposit, Redwood Falls, Minnesota: Unpublished report of the International Minerals and Chemical Corporation, Industrial Minerals Division, Geology Department, 14 p. text; various unnumbered tables and appendices; 4 pls.

Winchell, N.H.. 1874, The geology of the Minnesota valley: Minnesota Geological and Natural History Survey Annual Report, 2nd, for the year 1873, p. 127-212. 





\section{Periodicals}

Earthquakes \& Volcanoes (issued bimonthly).

Preliminary Determination of Epicenters (issued monthly).

\section{Technical Books and Reports}

Professional Papers are mainly comprehensive scientific reports of wide and lasting interest and importance to professional scientists and engineers. Included are reports on the results of resource studies and of topographic, hydrologic, and geologic investigations. They also include collections of related papers addressing different aspects of a single scientific topic.

Bulletins contain significant data and interpretations that are of lasting scientific interest but are generally more limited in scope or geographic coverage than Professional Papers. They include the results of resource studies and of geologic and topographic investigations; as well as collections of short papers related to a specific topic.

Water-Supply Papers are comprehensive reports that present significant interpretive results of hydrologic investigations of wide interest to professional geologists, hydrologists, and engineers. The series covers investigations in all phases of hydrology, including hydrology, availability of water, quality of water, and use of water.

Circulars present administrative information or important scientific information of wide popular interest in a format designed for distribution at no cost to the public. Information is usually of short-term interest.

Water-Resources Investigations Reports are papers of an interpretive nature made available to the public outside the formal USGS publications series. Copies are reproduced on request unlike formal USGS publications, and they are also available for public inspection at depositories indicated in USGS catalogs.

Open-File Reports include unpublished manuscript reports, maps, and other material that are made available for public consultation at depositories. They are a nonpermanent form of publication that may be cited in other publications as sources of information.

\section{Maps}

Geologic Quadrangle Maps are multicolor geologic maps on toJographic bases in $71 / 2$ - or 15 -minute quadrangle formats (scales mainy 1:24,000 or 1:62,500) showing bedrock, surficial, or engineering ggeology. Maps generally include brief texts; some maps include structure: and columnar sections only.

Geophysical Investigations Maps are on topographic or planimetic bases at various scales, they show results of surveys using geophysi:al techniques, such as gravity, magnetic, seismic, or radioactivity, which eflect subsurface structures that are of economic or geologic signifieance. Many maps include correlations with the geology.

Miscellaneous Investigations Series Maps are on planimetric or opographic bases of regular and irregular areas at various scales; they mresent a wide variety of format and subject matter. The series also in:ludes 7 1/2-minute quadrangle photogeologic maps on planimetric ases which show geology as interpreted from aerial photographs. The eries also includes maps of Mars and the Moon.
Coal Investigations Maps are geologic maps on topographic or planimetric bases at various scales showing bedrock or surficial geology, stratigraphy, and structural relations in certain coal-resource areas.

Oil and Gas Investigations Charts show stratigraphic information for certain oil and gas fields and other areas having petroleum potential.

Miscellaneous Field Studies Maps are multicolor or black-andwhite maps on topographic or planimetric bases on quadrangle or irregular areas at various scales. Pre-1971 maps show bedrock geology in relation to specific mining or mineral-deposit problems; post-1971 maps are primarily black-and-white maps on various subjects such as environmental studies or wilderness mineral investigations.

Hydrologic Investigations Atlases are multicolored or black-andwhite maps on topographic or planimetric bases presenting a wide range of geohydrologic data of both regular and irregular areas; the principal scale is 1:24,000, and regional studies are at 1:250,000 scale or smaller.

\section{Catalogs}

Permanent catalogs, as well as some others, giving comprehensive listings of U.S. Geological Survey publications are available under the conditions indicated below from USGS Map Distribution, Box 25286, Building 810, Denver Federal Center, Denver, CO 80225. (See latest Price and Availability List.)

"Publications of the Geological Survey, 1879-1961" may be purchased by mail and over the counter in paperback book form and as a set microfiche.

"Publications of the Geological Survey, 1962-1970" may be purchased by mail and over the counter in paperback book form and as a set of microfiche.

"Publications of the U.S. Geological Survey, 1971-1981" may be purchased by mail and over the counter in paperback book form (two volumes, publications listing and index) and as a set of microfiche.

Supplements for 1982, 1983, 1984, 1985, 1986, and for subsequent years since the last permanent catalog may be purchased by mail and over the counter in paperback book form.

State catalogs, "List of U.S. Geological Survey Geologic and Water-Supply Reports and Maps For (State)," may be purchased by mail and over the counter in paperback booklet form only.

"Price and Availability List of U.S. Geological Survey Publications," issued annually, is available free of charge in paperback booklet form only.

Selected copies of a monthly catalog "New Publications of the U.S. Geological Survey" is available free of charge by mail or may be obtained over the counter in paperback booklet form only. Those wishing a free subscription to the monthly catalog "New Publications of the U.S. Geological Survey" should write to the U.S. Geological Survey, $582 \mathrm{Na}-$ tional Center, Reston, VA 22092.

Note.-Prices of Government publications listed in older catalogs, announcements, and publications may be incorrect. Therefore, the prices charged may differ from the prices in catalogs, announcements, and publications. 
